\title{
SHORT-TIME EXISTENCE OF SOLUTIONS FOR MEAN-FIELD GAMES WITH CONGESTION
}

\author{
DIOGO A. GOMES AND VARDAN K. VOSKANYAN
}

\begin{abstract}
We consider time-dependent mean-field games with congestion that are given by a system of a Hamilton-Jacobi equation coupled with a Fokker-Planck equation. The congestion effects make the Hamilton-Jacobi equation singular. These models are motivated by crowd dynamics where agents have difficulty moving in high-density areas. Uniqueness of classical solutions for this problem is well understood. However, existence of classical solutions, was only known in very special cases - stationary problems with quadratic Hamiltonians and some time-dependent explicit examples. Here, we prove short-time existence of $C^{\infty}$ solutions in the case of sub-quadratic Hamiltonians.
\end{abstract}

\section{INTRODUCTION}

Here, we study the time-dependent mean-field games with congestion given by the system

$$
\left\{\begin{array}{l}
-u_{t}-\Delta u+m^{\alpha} H_{0}\left(x, \frac{D u}{m^{\alpha}}\right)+b \cdot D u=V(x, m(x, t)), \\
m_{t}-\Delta m-\operatorname{div}\left(D_{p} H_{0}\left(x, \frac{D u}{m^{\alpha}}\right) m\right)-\operatorname{div}(b m)=0 \\
u(x, T)=\Psi(x), m(x, 0)=m_{0}(x)
\end{array}\right.
$$

Because we work in the spatially periodic setting, the variable $x$ takes values on the $d$-dimensional torus $\mathbb{T}^{d}$. The unknowns in (10) are the functions $u: \mathbb{T}^{d} \times[0, T] \rightarrow \mathbb{R}$, and $m: \mathbb{T}^{d} \times[0, T] \rightarrow \mathbb{R}^{+}$. The functions $H_{0}: \mathbb{T}^{d} \times \mathbb{R}^{d} \rightarrow \mathbb{R}, V: \mathbb{T}^{d} \times \mathbb{R} \rightarrow \mathbb{R}$, and $b, \Psi, m_{0}: \mathbb{T}^{d} \rightarrow \mathbb{R}$ are given $C^{\infty}$ functions with $m_{0}>0$. Moreover, $V(x, m)$ is increasing in $m$. Detailed hypotheses on $H_{0}, b, V, \Psi$ and $m_{0}$ are presented in Section 2. A concrete Hamiltonian $H_{0}$ for which our results apply is the following: for $\gamma \in(1,2)$, set $\frac{1}{\gamma}+\frac{1}{\gamma^{\prime}}=1$. Consider the Lagrangian:

$$
L_{0}(x, v)=a(x)\left(1+|v|^{2}\right)^{\frac{\gamma^{\prime}}{2}},
$$

where $a \in \mathcal{C}^{\infty}\left(\mathbb{T}^{d}\right), a>0$. Define $H_{0}$ as the Legendre transform of $L_{0}$ :

$$
H_{0}(x, p)=\sup _{v}\left[-v \cdot p-L_{0}(x, v)\right] .
$$

The uniqueness of solutions to (1) was proven in 31] (see also [24]) under Assumptions 10 . 12 of Section 2 Here, we prove the existence of smooth solutions for small terminal times and sub-quadratic Hamiltonians:

Theorem 1. Under Assumptions 1 12, (cf. Section 2), there exists a time $T_{0}>0$ such that for any terminal time $T \leq T_{0}$ there exists a $C^{\infty}$ solution $(u, m)$ to (1), with $m>0$.

Mean-field games have become an important research field since the seminal works of J-M. Lasry and P-L. Lions [28, 29, 30, and M. Huang, P. Caines and R. Malhamé 26, 25. Diverse questions have been studied intensively, these include stationary mean-field games [18, 17, 12, classical and weak solutions for time-dependent problems, see, respectively, [16, 15, 13, 14, and [33, 34, 2], finite state models [9, 10, 7, 6, 21, 20], extended mean-field games [22, and obstacle

Date: December 2, 2018.

2010 Mathematics Subject Classification. 35J47, 35A01.

Key words and phrases. Mean Field Game; Congestion models.

D. Gomes and V. Voskanyan were partially supported by KAUST baseline and start-up funds and KAUST SRI, Center for Uncertainty Quantification in Computational Science and Engineering. 
problems [11]. For a recent survey, see [19]. Congestion problems were addressed initially by P-L. Lions 31, who proved the uniqueness of smooth enough solutions. Two alternative approaches to congestion problems are density constraints, introduced in [35, 32, and nonlinear mobilities, see 1]. A recent existence result, see [8], regards the stationary congestion problem with quadratic costs. The existence of solutions in the time-dependent setting has not been established previously.

Before proceeding, we briefly discuss the motivation for (1). We consider a large population of agents on $\mathbb{T}^{d}$, whose statistical evolution over time is encoded in an unknown probability density $m(x, t)$. Let $\left(\Omega, \mathcal{F}_{t}, P\right)$ be a filtered probability space supporting a $d$-dimensional Brownian motion $W_{t}$. Let $\mathbb{E}$ be the expected value operator. Consider an agent whose location at time $t$ is $x$. The cost function for this agent, sometimes called value or utility function, is

$$
u(x, t)=\inf _{v} \mathbb{E} \int_{t}^{T} L\left(X_{s}, v_{s}, m\left(X_{s}, s\right), s\right) d s+\Psi\left(X_{T}\right),
$$

where the trajectory $X$ controlled by the dynamics

$$
d X_{s}=v_{s} d s+\sqrt{2} d W_{s}, X_{0}=x,
$$

and the infimum is taken over bounded $\mathcal{F}_{t}$-progressively measurable controls $v_{s}$. Here, $\Psi: \mathbb{T}^{d} \rightarrow \mathbb{R}^{- \text {a }}$ is the terminal cost. The Lagrangian $L$ has the form

$$
L(x, v, m, t)=m^{\alpha} L_{0}(x, v-b(x, t))+V(x, m) .
$$

Detailed assumptions on $L_{0}$ are given in the next Section. The constant $\alpha$ determines the strength of the congestion effects. These are encoded in the term $m^{\alpha} L_{0}(x, v-b(x, t))$ that makes it more expensive to move in regions of high density if the drift $v$ is substantially different from a reference vector field $b: \mathbb{T}^{d} \times[0, T] \rightarrow \mathbb{R}^{d}$. Finally, the function $V: \mathbb{T}^{d} \times \mathbb{R}_{0}^{+} \rightarrow \mathbb{R}$ accounts for other spatial preferences of the agents.

The Hamiltonian is the Legendre transform of $L$, given by

$$
H(x, p, m, t)=\sup _{v}\{-v \cdot p-L(x, v, m, t)\}=m^{\alpha} H_{0}\left(x, \frac{p}{m^{\alpha}}\right)+b(x, t) \cdot p,
$$

where $H_{0}$ is the Legendre transform of $L_{0}$. Under standard assumptions regarding rationality and symmetry, the mean-field problem that models this setup is (11). It comprises a system of a second-order Hamilton-Jacobi equation for the value function $u$ coupled with a KolmogorovFokker-Planck equation for the density of agents $m$.

We conclude this introduction with the structure of the paper: in Section 2 , we state the main assumptions used in this manuscript. Afterwards, in Section 3, we discuss various estimates that hold for arbitrary values of the terminal time $T$. Then, in Section 4 , we present a new technique to address the short-time problem by controlling the growth of $\frac{1}{m}$. Next, in Section 5 we establish further regularity for the solutions. Section 6 concludes the paper with the proof of Theorem 1.

\section{Assumptions}

Throughout the present manuscript, we work under several hypotheses that we state next. Assumptions 1 and 2 concern the smoothness of the initial and terminal data, and the various functions in (11). Here, we work with $C^{\infty}$ data to simplify the arguments. However, it would be possible to carry out the proofs with less regularity, and obtain the existence of solutions with $C^{k}$ regularity for $k$ large enough. Assumptions 3,7] and 10] are standard hypotheses in optimal control, viscosity solutions, and mean-field games. They are stated explicitly for the convenience and clarity of the paper and do not result in a substantial loss of generality. A model Hamiltonian that satisfies those is (3). Assumptions 8 and 9 are specific to the present problem and impose, respectively, a bound on the congestion exponent and subquadratic growth for the Hamiltonian. Subquadratic Hamiltonians correspond to superquadratic Lagrangians. In the example (3), this is reflected in the condition $\frac{1}{\gamma}+\frac{1}{\gamma^{\prime}}=1$ satisfied by the exponent in (2). Finally, Assumptions 11 and 12 are required for the uniqueness, see 31 . 
Assumption 1. The terminal cost $\Psi: \mathbb{T}^{d} \rightarrow \mathbb{R}$, the reference velocity $b: \mathbb{T}^{d} \times[0, T] \rightarrow \mathbb{R}^{d}$ and the potential $V: \mathbb{T}^{d} \times \mathbb{R} \rightarrow \mathbb{R}$ are $C^{\infty}$ functions, globally bounded with bounded derivatives of all orders.

Assumption 2. The initial distribution $m_{0}: \mathbb{T}^{d} \rightarrow \mathbb{R}$ is a $C^{\infty}$ probability density: $\int_{\mathbb{T}^{d}} m_{0}(x) d x=$ 1. Moreover, there exists $k_{0}>0$, such that $m_{0}(x) \geq k_{0}$ for all $x \in \mathbb{T}^{d}$.

Assumption 3. The Lagrangian $L_{0}: \mathbb{T} \times \mathbb{R}^{d}$ is $C^{\infty}$, and the map

$$
v \mapsto L_{0}(x, v)
$$

is strictly convex for every $x \in \mathbb{T}^{d}$.

Assumption 4. $L_{0}$ is positive: $L_{0}(x, v) \geq 0, \forall(x, v) \in \mathbb{T}^{d} \times \mathbb{R}^{d}$.

Assumption 5. There exists conjugated powers $\gamma, \gamma^{\prime}>1, \frac{1}{\gamma}+\frac{1}{\gamma^{\prime}}=1$, and constants $C_{i}, c_{i}>$ $0, i=1,2$ such that

$$
C_{1} \frac{|v|^{\gamma^{\prime}}}{\gamma^{\prime}}-c_{1} \leq L_{0}(x, v) \leq C \frac{|v|^{\gamma^{\prime}}}{\gamma^{\prime}}+c, \forall x \in \mathbb{T}^{d}, v \in \mathbb{R}^{d} .
$$

Remark 1. The definition of Legendre transform implies the convexity of $H_{0}$. Thus, we have

$$
H_{0}(x, p)-p \cdot D_{p} H_{0}(x, p) \leq H_{0}(x, 0)=\sup _{v}\left\{-L_{0}(x, v)\right\} \leq 0,
$$

using Assumption 4 .

Remark 2. Under Assumption [, the Hamiltonian $H_{0}: \mathbb{T}^{d} \times \mathbb{R}^{d} \rightarrow \mathbb{R}$ is $C^{\infty}$.

Remark 3. Let $H_{0}(x, p)=\sup _{v}\left\{-p \cdot v-L_{0}(x, v)\right\}$ be the Legendre transform of $L_{0}$. Then, the Assumptions 3 [5 imply

$$
C_{1}^{\prime} \frac{|p|^{\gamma}}{\gamma}-c_{1}^{\prime} \leq H_{0}(x, p) \leq C_{2}^{\prime} \frac{|p|^{\gamma}}{\gamma}+c_{2}^{\prime}, \quad \forall x \in \mathbb{T}^{d}, p \in \mathbb{R}^{d} .
$$

Assumption 6. There exist positive constants $c, C>0$ such that

$$
p \cdot D_{p} H_{0}(x, p)-H_{0}(x, p) \geq c|p|^{\gamma}-C .
$$

Assumption 7. There exists a constant $C$ such that

$$
\left|D_{p} H_{0}(x, p)\right| \leq C|p|^{\gamma-1}+C .
$$

Remark 4. By combining Remark 3 with the Assumption 6 , we conclude that there exist positive constants $c, C$ such that, for any $r>1$,

$$
c|p|^{\gamma} \leq H_{0}(x, p)+r p \cdot D_{p} H_{0}(x, p)+C r .
$$

Assumption 8. The exponent $\alpha$ in the congestion term $\left(m^{\alpha}\right)$ satisfies the inequality $0 \leq \alpha<\frac{2}{d-2}$.

Assumption 9. $H_{0}$ has sub-quadratic growth, i.e. $\gamma<2$.

The next three assumptions are required for the uniqueness of solutions.

Assumption 10. The Hamiltonian $H_{0}$ is $C^{\infty}$, and the map

$$
p \mapsto H_{0}(x, p)
$$

is strictly convex for every $x \in \mathbb{T}^{d}$, that is, $D_{p p}^{2} H_{0}(x, p)>0, \forall(x, p) \in \mathbb{T}^{d} \times \mathbb{R}^{d}$.

Remark 5. The previous Assumption implies that $H_{0}$ is uniformly convex on compacts, i.e., for any $R>0$, there exists $\theta_{R}>0$ such that $D_{p p}^{2} H_{0}(x, p) \geq \theta_{R} I, \forall(x, p) \in \mathbb{T}^{d} \times \mathbb{R}^{d}$, with $|p| \leq R$.

Assumption 11. For $p \neq 0$, the following inequality holds:

$$
D_{p} H_{0}(x, p) \cdot p-H_{0}(x, p)>\frac{\alpha}{4} p^{t} \cdot D_{p p} H_{0}(x, p) \cdot p .
$$

Assumption 12. The potential $V: \mathbb{T}^{d} \times \mathbb{R} \rightarrow \mathbb{R}$ is strictly increasing in the second variable. 
For the Hamiltonian $H_{0}$ given by (3), Assumption [10 is satisfied for every $\gamma>1$. A simple calculation shows that Assumption 11 holds if $\alpha<\frac{4\left(\gamma^{\prime}-1\right)}{\gamma^{\prime}}=\frac{4}{\gamma}$. A potential $V$ for which Assumption 12 is valid is $V(x, z)=\arctan (z)$.

\section{ESTIMATES FOR ARBITRARY TERMINAL TIME}

The main result of this paper is the existence of smooth solutions to (1) for small terminal time $T$. Nevertheless, various estimates we need are valid for arbitrary $T$. We report those in this section.

We begin with an auxiliary Lemma

Lemma 1. For $0 \leq \tau \leq T$, and $\phi \in C^{\infty}\left(\mathbb{T}^{d}\right), \phi \geq 0$ with $\|\phi\|_{L^{1}\left(\mathbb{T}^{d}\right)} \leq 1$, let $\rho$ be the solution to

$$
\left\{\begin{array}{l}
\rho_{t}=\Delta \rho \\
\rho(x, \tau)=\phi(x)
\end{array}\right.
$$

Denote by $2^{*}$ the Sobolev conjugate exponent of 2 , given by $\frac{1}{2^{*}}=\frac{1}{2}-\frac{1}{d}$. Then, for any $q$ with $1<q<\frac{2^{*}}{2}$, there exists a constant $C_{q}$ such that

$$
\|\rho\|_{L^{1}\left(L^{q}(d x), d t\right)}=\int_{\tau}^{T}\left(\int_{\mathbb{T}^{d}} \rho^{q} d x\right)^{\frac{1}{q}} d t \leq C_{q} .
$$

Proof. By the maximum principle, $\rho \geq 0$. Furthermore, $\frac{d}{d t} \int_{\mathbb{T}^{d}} \rho(x, t) d x=0$. In particular, for any $t \geq \tau\|\rho(\cdot, t)\|_{L^{1}\left(\mathbb{T}^{d}\right)}=\|\phi\|_{L^{1}\left(\mathbb{T}^{d}\right)} \leq 1$. Multiplying the heat equation (5) by $\rho^{\delta-1}$, for $0<\delta<1$, and integrating by parts, we get

$$
c_{\delta} \int_{\tau}^{T} \int_{\mathbb{T}^{d}}\left|D\left(\rho^{\frac{\delta}{2}}\right)\right|^{2} d x d t=\frac{1}{\delta} \int_{\mathbb{T}^{d}}\left(\rho^{\delta}(x, T)-\rho^{\delta}(x, \tau)\right) d x \leq \frac{1}{\delta},
$$

for any $\varepsilon>0$, where $c_{\delta}=\frac{4(1-\delta)}{\delta^{2}}$. Here, we used Jensen's inequality to obtain

$$
0 \leq \int_{\mathbb{T}^{d}} \rho^{\delta}(x, \tau) d x, \quad \int_{\mathbb{T}^{d}} \rho^{\delta}(x, T) d x \leq 1
$$

From the Gagliardo-Nirenberg inequality,

$$
\left\|\rho^{\frac{\delta}{2}}(\cdot, t)\right\|_{L^{p_{\delta}\left(\mathbb{T}^{d}\right)}} \leq C\left\|D\left(\rho^{\frac{\delta}{2}}\right)(\cdot, t)\right\|_{L^{2}\left(\mathbb{T}^{d}\right)}^{\delta}\left\|\rho^{\frac{\delta}{2}}(\cdot, t)\right\|_{L^{2}\left(\mathbb{T}^{d}\right)}^{1-\delta},
$$

where $\frac{1}{p_{\delta}}=\left(\frac{1}{2}-\frac{1}{d}\right) \delta+\frac{1-\delta}{2}$. Since $\left\|\rho^{\frac{\delta}{2}}(\cdot, t)\right\|_{L^{2}\left(\mathbb{T}^{d}\right)} \leq\|\rho(\cdot, t)\|_{L^{1}\left(\mathbb{T}^{d}\right)}^{\frac{\delta}{2}} \leq 1$, we have

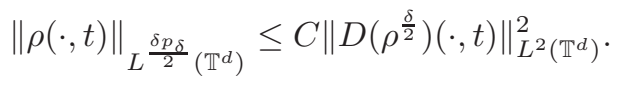

Finally, integrating the previous estimate in time and using ([6) , we obtain $\|\rho\|_{L^{1}\left(L^{\frac{\delta p_{\delta}}{2}}(d x), d t\right)} \leq C_{\delta}$. To end the proof, we observe that $p_{\delta} \rightarrow 2^{*}$ when $\delta \rightarrow 1$.

Proposition 1. Under Assumptions 1 1 4 there exists a constant $C:=C\left(\|V\|_{\infty},\|\psi\|_{\infty}, T\right)$ such that for any $C^{\infty}$ solution $(u, m)$ of (1), we have $\|m(\cdot, t)\|_{L^{1}\left(\mathbb{T}^{d}\right)}=1, \forall 0 \leq t \leq T$, and, additionally, $u \geq-C$.

Proof. Integrating the second equation we have $\left(\int_{\mathbb{T}^{d}} m(x, t) d x\right)_{t}=0$. Therefore, $\int_{\mathbb{T}^{d}} m(x, t) d x=$ 1 , for all $t \geq 0$. To prove the upper bound for $u$, we apply the nonlinear adjoint method [5] (for further applications, see also [36]). Let $\zeta$ be a solution to

$$
\left\{\begin{array}{l}
\zeta_{t}-\operatorname{div}\left(D_{p} H_{0}\left(x, \frac{D u}{m^{\alpha}}\right) \zeta\right)-\operatorname{div}(b \zeta)=\Delta \zeta \\
\zeta(x, \tau)=\phi(x)
\end{array}\right.
$$


with $\phi \in C^{\infty}\left(\mathbb{T}^{d}\right), \phi \geq 0$. We multiply the first equation in (11) by $\zeta$ and subtract (7) multiplied by $u$. Then, we integrate by parts and gather

$$
-\left[\int_{\mathbb{T}^{d}} u \zeta d x\right]_{t}+\int_{\mathbb{T}^{d}} m^{\alpha}\left[H_{0}\left(x, \frac{D u}{m^{\alpha}}\right)-\frac{D u}{m^{\alpha}} D_{p} H_{0}\left(x, \frac{D u}{m^{\alpha}}\right)\right] \zeta d x=\int_{\mathbb{T}^{d}} V \zeta d x .
$$

Integrating on $[\tau, T]$ and using (4), we obtain

$$
-\int_{\mathbb{T}^{d}} \Psi(x) \zeta(x, T) d x+\int_{\mathbb{T}^{d}} u(x, \tau) \phi(x) d x \geq \int_{\tau}^{T} \int_{\mathbb{T}^{d}} V \zeta d x d t .
$$

By the maximum principle, $\zeta \geq 0$, because $\phi \geq 0$. Integrating (7) with respect to $x$, we get $\int \zeta d x=\int \phi d x$. This identity, together with the above inequality, yields

$$
\int_{\mathbb{T}^{d}} u(x, \tau) \phi(x) d x \geq-\left[(T-\tau)\|V\|_{\infty}+\|\psi\|_{\infty}\right]\|\phi\|_{L^{1}\left(\mathbb{T}^{d}\right)} .
$$

Since this estimate holds for every $C^{\infty} \phi \geq 0$, we obtain $-u(x, \tau) \leq(T-\tau)\|V\|_{\infty}+\|\psi\|_{\infty}$.

Proposition 2. Under Assumptions [1]6, there exists a constant $C:=C\left(\|V\|_{\infty},\|\psi\|_{\infty}, T\right)$ such that for any $C^{\infty}$ solution $(u, m)$ to (1), we have

$$
\int_{0}^{T} \int_{\mathbb{T}^{d}} \frac{|D u|^{\gamma}}{m^{\bar{\alpha}}} d x d t \leq C
$$

and

$$
\int_{\mathbb{T}^{d}}|u(x, t)| d x \leq C, t \in[0, T]
$$

where

$$
\bar{\alpha}=(\gamma-1) \alpha<1 .
$$

Proof. We integrate the first equation in (1) with respect to $x$ and $t$. Then, we use the bounds on $u$ from the previous proposition, to get

$$
\int_{t}^{T} \int_{\mathbb{T}^{d}}\left(m^{\alpha} H_{0}\left(x, \frac{D u}{m^{\alpha}}\right)+b \cdot D u\right) d x d s=\int_{\mathbb{T}^{d}} u(x, T) d x-\int_{\mathbb{T}^{d}} u(x, t) d x+\int_{t}^{T} \int_{\mathbb{T}^{d}} V d x d s \leq C .
$$

By Remark 3, $|p|^{\gamma} \leq C\left(H_{0}(x, p)+b \cdot p\right)+C$. Accordingly,

$$
\begin{array}{rl}
\int_{\mathbb{T}^{d}} & u(x, t) d x+\int_{t}^{T} \int_{\mathbb{T}^{d}} \frac{|D u|^{\gamma}}{m^{\bar{\alpha}}} d x d t \\
& \leq C \int_{t}^{T} \int_{\mathbb{T}^{d}} m^{\alpha}\left(H_{0}\left(x, \frac{D u}{m^{\alpha}}\right)+b \cdot D u\right) d x d s+C \int_{t}^{T} \int_{\mathbb{T}^{d}} m^{\alpha} d x d s \leq C .
\end{array}
$$

This inequality, combined with the lower bound on $u$ of Proposition 1 yields (8). Moreover, since

$$
\int u \leq C
$$

using again the lower bound on $u$ of Proposition 1, we obtain (9).

Proposition 3. Under Assumptions [1]6, there exists a constant $C:=C\left(\|V\|_{\infty},\|\psi\|_{\infty}, T\right)$ such that for any $C^{\infty}$ solution $(u, m)$ to (1) we have

$$
\int_{0}^{t} \int_{\mathbb{T}^{d}}|D u|^{\gamma} m^{1-\bar{\alpha}} d x d t \leq C+C \int_{0}^{t} \int_{\mathbb{T}^{d}} m^{1+\alpha} d x d t
$$

for all $0 \leq t \leq T$, where $\bar{\alpha}$ is given by (10). 
Proof. We multiply the first equation in (10) by $m$ and subtract the second equation multiplied by $u$. Then, integration by parts yields:

$$
\begin{aligned}
& \int_{0}^{t} \int_{\mathbb{T}^{d}} m^{1+\alpha}\left[\frac{D u}{m^{\alpha}} D_{p} H_{0}\left(x, \frac{D u}{m^{\alpha}}\right)-H_{0}\left(x, \frac{D u}{m^{\alpha}}\right)\right] d x d t=-\int_{0}^{t} \int_{\mathbb{T}^{d}} V m d x+\int_{\mathbb{T}^{d}} u(x, 0) m_{0}(x) d x \\
& -\int_{\mathbb{T}^{d}} u(x, t) m(x, t) d x \leq C+\left\|m_{0}\right\|_{\infty} \int_{\mathbb{T}^{d}}|u(x, 0)| d x-\inf _{x} u(x, t) \leq C,
\end{aligned}
$$

where the last inequality follows from lower bounds on $u$ from Proposition 1, and the bound on $\int_{\mathbb{T}^{d}}|u| d x$ in the previous Proposition. The claim in the statement follows from Assumption 6 by using the inequality $p D_{p} H_{0}(x, p)-H_{0}(x, p) \geq c|p|^{\gamma}-C$, for some $c, C>0$.

Proposition 4. Under Assumptions 1 17, there exists a constant $C:=C\left(\|V\|_{\infty},\|\psi\|_{\infty}, T\right)$ such that for any $C^{\infty}$ solution $(u, m)$ of (1), we have

$$
\int_{\mathbb{T}^{d}} m^{1+\alpha}(x, t) d x+\int_{0}^{t} \int_{\mathbb{T}^{d}} m^{\alpha-1}(x, s)|D m(x, s)|^{2} d x d s \leq C .
$$

Proof. We begin by multiplying the second equation by $(\alpha+1) m^{\alpha}$. Next, integrating by parts, we conclude

$$
\begin{aligned}
& {\left[\int_{\mathbb{T}^{d}} m^{1+\alpha}(x, t) d x\right]_{t}=-\alpha(1+\alpha) \int_{\mathbb{T}^{d}} m^{\alpha-1}\left[|D m|^{2}+m D_{p} H_{0}\left(x, \frac{D u}{m^{\alpha}}\right) \cdot D m+m b \cdot D m\right] d x} \\
& \leq-\alpha(1+\alpha) \int_{\mathbb{T}^{d}}\left[\frac{1}{2} m^{\alpha-1}|D m|^{2}-\left|D_{p} H_{0}\left(x, \frac{D u}{m^{\alpha}}\right)\right|^{2} m^{\alpha+1}-\|b\|_{\infty}^{2} m^{\alpha+1}\right] d x \\
& \leq-\alpha(1+\alpha) \int_{\mathbb{T}^{d}}\left[\frac{1}{2} m^{\alpha-1}|D m|^{2}-C|D u|^{2(\gamma-1)} m^{\alpha+1-2 \bar{\alpha}}-\|b\|_{\infty}^{2} m^{\alpha+1}\right] d x \\
& \leq-\alpha(1+\alpha) \int_{\mathbb{T}^{d}}\left[\frac{1}{2} m^{\alpha-1}|D m|^{2}-C|D u|^{\gamma} m^{1-\bar{\alpha}}-C m^{\alpha+1}\right] d x,
\end{aligned}
$$

where, in the last inequality, we have used Young's inequality:

$$
|p|^{2(\gamma-1)} m^{1+\alpha-2 \bar{\alpha}}=\left(|p|^{\gamma} m^{1-\bar{\alpha}}\right)^{\frac{2(\gamma-1)}{\gamma}}\left(m^{1+\alpha}\right)^{\frac{2-\gamma}{\gamma}} \leq \frac{2(\gamma-1)}{\gamma}|p|^{\gamma} m^{1-\bar{\alpha}}+\frac{2-\gamma}{\gamma} m^{1+\alpha}
$$

and the definition of $\bar{\alpha}$ in (10). Integrating (11) from 0 to $t$ and using Proposition 3 , we conclude that

$$
\int_{\mathbb{T}^{d}} m^{1+\alpha}(x, t) d x+\int_{0}^{t} \int_{\mathbb{T}^{d}} m^{\alpha-1}(x, s)|\operatorname{Dm}(x, s)|^{2} d x d s \leq C+C \int_{0}^{t} \int_{\mathbb{T}^{d}} m^{1+\alpha} d x d t .
$$

In particular,

$$
\int_{\mathbb{T}^{d}} m^{1+\alpha}(x, t) d x \leq C+C \int_{0}^{t} \int_{\mathbb{T}^{d}} m^{1+\alpha} d x d t
$$

Thus, by Gronwall's inequality, we have $\int_{\mathbb{T}^{d}} m^{1+\alpha}(x, t) d x \leq C$. This estimate combined with (12) yields

$$
\int_{0}^{t} \int_{\mathbb{T}^{d}} m^{\alpha-1}(x, s)|D m(x, s)|^{2} d x d s \leq C .
$$

Corollary 1. Under Assumptions 1] there exists a constant $C:=C\left(\|V\|_{\infty},\|\psi\|_{\infty}, T\right)$ such that

$$
\int_{0}^{t} \int_{\mathbb{T}^{d}}|D u|^{\gamma} m^{1-\bar{\alpha}} d x d t \leq C
$$

Proof. The Corollary follows by combining Proposition 3 with Proposition 4 
Proposition 5. Under Assumptions [1]8, there exists a constant $C:=C\left(\|V\|_{\infty},\|\psi\|_{\infty}, T-t\right)$ such that, for any $C^{\infty}$ solution $(u, m)$ of (1),$u \leq C$.

Proof. Let $\rho$ be as in Lemma 1. Multiplying the first equation in (1) by $\rho$, subtracting the equation for $\rho$ multiplied by $u$, and integrating by parts, we gather

$$
-\left[\int_{\mathbb{T}^{d}} u \rho d x\right]_{t}+\int_{\mathbb{T}^{d}} m^{\alpha}\left[H_{0}\left(x, \frac{D u}{m^{\alpha}}\right)+b \cdot \frac{D u}{m^{\alpha}}\right] \rho d x=\int_{\mathbb{T}^{d}} V \rho d x,
$$

where we used the fact that $H_{0}(x, p)+b \cdot p$ is bounded by below as a consequence of Remark 3 Hence, integrating in time, we conclude

$$
\int_{\mathbb{T}^{d}} u(x, \tau) \phi(x) d x \leq \int_{\tau}^{T} \int_{\mathbb{T}^{d}} V \rho d x d t+\int_{\mathbb{T}^{d}} \Psi(x) \rho(x, T) d x+C \int_{\tau}^{T} \int_{\mathbb{T}^{d}} m^{\alpha} \rho d x d t .
$$

Using Holder's inequality and the bounds $\int_{\mathbb{T}^{d}} \rho(x, t) d x \leq 1, \int_{\mathbb{T}^{d}} m^{1+\alpha}(x, t) d x \leq C$, we get

$$
u(x, \tau) \leq(T-\tau)\|V\|_{\infty}+\|\Psi\|_{\infty}+C\|\rho\|_{L^{1}\left(L^{1+\alpha}(d x), d t\right)} .
$$

Because Assumption 8 holds, $\alpha<\frac{2}{d-2}$. Consequently $1+\alpha<\frac{2^{*}}{2}$. Therefore, we can apply Lemma 1 to prove the result.

Corollary 2. Under Assumptions 10, there exists a constant $C:=C\left(\|V\|_{\infty},\|\psi\|_{\infty}, T-t\right)$ such that for any $C^{\infty}$ solution $(u, m)$ of (11) $\|u\|_{L^{\infty}\left(\mathbb{T}^{d}\right)} \leq C$.

Proof. The result follows by combining Propositions 1 and 5 ,

Proposition 6. Under Assumptions 1 1, there exists constant $c_{r}, C_{r}=C_{r}(\alpha, T)>0$ that have polynomial growth in $r$, such that for any $C^{\infty}$ solution $(u, m)$ of (1) and $r>1$

$$
\int_{\mathbb{T}^{d}} \frac{1}{m^{r}(x, t)} d x+c_{r} \int_{0}^{t} \int_{\mathbb{T}^{d}}\left|D \frac{1}{m^{r / 2}}\right|^{2} d x d t+\int_{0}^{t} \int_{\mathbb{T}^{d}} \frac{|D u|^{\gamma}}{m^{r+\bar{\alpha}}} d x d t \leq C_{r}+C_{r} \int_{0}^{t} \int_{\mathbb{T}^{d}} \frac{1}{m^{q}} d x d t, \forall t,
$$

where $\bar{\alpha}$ is given by (10) and

$$
q=r+\frac{2 \bar{\alpha}}{2-\gamma} .
$$

Proof. By adding a constant to $u_{0}$, we can assume, without loss of generality, that $u \leq-1$. Fix $r>1$. We begin by multiplying the first equation in (1D) by $\frac{1}{m^{r}}$, and adding it to the second equation multiplied by $r \frac{u}{m^{r+1}}$. After integrating by parts, we obtain

$$
\begin{aligned}
& -\int_{\mathbb{T}^{d}}\left(\frac{u}{m^{r}}\right)_{t} d x-\int_{\mathbb{T}^{d}} r(r+1) \frac{u|D m|^{2}}{m^{r+2}} d x+\int_{\mathbb{T}^{d}} m^{\alpha} \frac{H_{0}+r \frac{D u}{m^{\alpha}} D_{p} H_{0}}{m^{r}} d x \\
& -\int_{\mathbb{T}^{d}} r(r+1) \frac{u D_{p} H_{0} D m}{m^{r+1}} d x+\int_{\mathbb{T}^{d}}\left[(r+1) \frac{b \cdot D u}{m^{r}}-r(r+1) u \frac{b D m}{m^{r+1}}-\frac{V}{m^{r}}\right] d x=0 .
\end{aligned}
$$

We integrate the inequality in $t$. For $m<1$, we have

whereas for $m \geq 1$

$$
\frac{|D u|}{m^{r}} \leq \epsilon \frac{|D u|^{\gamma}}{m^{r+\bar{\alpha}}}+\frac{1}{m^{r}}+C_{r, \epsilon},
$$

$$
\frac{|D u|}{m^{r}} \leq|D u| \leq|D u|^{\gamma} m^{1-\bar{\alpha}}+C .
$$

Taking into account these estimates and the bound in Corollary 1 we get

Then, we use the estimates:

$$
\int_{0}^{t} \int_{\mathbb{T}^{d}} \frac{|D u|}{m^{r}} \leq \int_{0}^{t} \int_{\mathbb{T}^{d}} \frac{|D u|^{\gamma}}{m^{r+\bar{\alpha}}}+\frac{1}{m^{r}}+\tilde{C}_{r, \epsilon} .
$$

$$
c \frac{|D u|^{\gamma}}{m^{r+\bar{\alpha}}} \leq m^{\alpha} \frac{H_{0}+r \frac{D u}{m^{\alpha}} D_{p} H_{0}}{m^{r}}+C \frac{r}{m^{r-\alpha}}(\text { see Remark } 4),
$$


and

to get

$$
\frac{|D m|}{m^{r+1}} \leq \epsilon \frac{|D m|^{2}}{m^{r+2}}+C_{\epsilon} \frac{1}{m^{r}}
$$

$$
\begin{aligned}
& \int_{\mathbb{T}^{d}} \frac{1}{m^{r}(x, t)} d x+c_{r} \int_{0}^{t} \int_{\mathbb{T}^{d}}\left|D \frac{1}{m^{r / 2}}\right|^{2} d x d s+c_{r} \int_{0}^{t} \int_{\mathbb{T}^{d}} \frac{|D u|^{\gamma}}{m^{r+\bar{\alpha}}} d x d s \leq \\
& C_{r} \int_{0}^{t} \int_{\mathbb{T}^{d}}|D u|^{\gamma-1} \frac{|D m|}{m^{r+\bar{\alpha}+1}} d x d s+C_{r} \int_{0}^{t} \int_{\mathbb{T}^{d}} \frac{1}{m^{r}} d x d s+C_{r} .
\end{aligned}
$$

The required estimate follows from the inequalities:

and

$$
|D u|^{\gamma-1} \frac{|D m|}{m^{r+\bar{\alpha}+1}} \leq \epsilon \frac{|D u|^{\gamma}}{m^{r+\bar{\alpha}}}+\epsilon \frac{|D m|^{2}}{m^{r+2}}+C_{\epsilon} \frac{1}{m^{q}},
$$

where $q$ is given by (14).

$$
\frac{1}{m^{r}} \leq \frac{1}{m^{q}}+1
$$

\section{Short-Time Estimates}

In this section, we establish estimates for $C^{\infty}$ solutions of (11) for small values of $T$. The key idea is to use the estimate in Proposition 6 to control the growth of $\frac{1}{m}$. Because $q>r$ in (14), we can only achieve bounds for small $T$. We begin with the following bound on $\frac{1}{m}$ :

Theorem 2. Under Assumptions 1] 9, there exist $r_{0}>0$, a time $t_{1}(r)>0$ and constants $C=$ $C(r, \gamma, \alpha)>0, \delta>0$, such that for any $C^{\infty}$ solution $(u, m)$ to (11) and $r \geq r_{0}$ :

$$
\int_{\mathbb{T}^{d}} \frac{1}{m^{r}(x, t)} d x \leq C\left[1+\frac{1}{\left(t_{1}-t\right)^{\delta}}\right], \forall t<t_{1} .
$$

Proof. We choose $r_{0}$ sufficiently large such that $\frac{2^{*}}{2} r=\frac{d r}{d-2}>q=r+\frac{2 \bar{\alpha}}{2-\gamma}$, for $r \geq r_{0}$. Let $\lambda>0$ be such that $\frac{2^{*}}{2} r \lambda+r(1-\lambda)=q$, that is, $\lambda=\frac{\bar{\alpha}(d-2)}{(2-\gamma) r}<1$, for $r \geq r_{0}$. We set $\bar{\lambda}=\frac{2^{*}}{2} \lambda=\frac{\bar{\alpha} d}{(2-\gamma) r}$. Provided $r_{0}$ is large enough, $\bar{\lambda}<1$ and $\beta=\frac{1-\bar{\lambda}}{1-\lambda}>1$, for all $r \geq r_{0}$. Then, using Hölder's and Young's inequalities, we obtain

$$
\begin{gathered}
\int_{\mathbb{T}^{d}} \frac{1}{m^{q}} d x \leq\left(\int_{\mathbb{T}^{d}} \frac{1}{m^{\frac{2^{*}}{2} r}} d x\right)^{\lambda}\left(\int_{\mathbb{T}^{d}} \frac{1}{m^{r}} d x\right)^{1-\lambda}=\left[\left(\int_{\mathbb{T}^{d}} \frac{1}{m^{\frac{2^{*}}{2}}} d x\right)^{2 / 2^{*}}\right]^{\bar{\lambda}}\left[\left(\int_{\mathbb{T}^{d}} \frac{1}{m^{r}} d x\right)^{\beta}\right]^{1-\bar{\lambda}} \leq \\
\varepsilon \bar{\lambda}\left(\int_{\mathbb{T}^{d}} \frac{1}{m^{\frac{2^{*}}{2} r}} d x\right)^{2 / 2^{*}}+\frac{1}{\varepsilon^{\tau}}(1-\bar{\lambda})\left(\int_{\mathbb{T}^{d}} \frac{1}{m^{r}} d x\right)^{\beta},
\end{gathered}
$$

for any $\varepsilon>0$ and some exponent $\tau>0$. From Sobolev's inequality,

$$
\int_{\mathbb{T}^{d}} \frac{|D m|^{2}}{m^{r+2}} d x=\frac{4}{r^{2}} \int_{\mathbb{T}^{d}}\left|D\left(\frac{1}{m^{r / 2}}\right)\right|^{2} d x \geq c \frac{4}{r^{2}}\left(\int_{\mathbb{T}^{d}} \frac{1}{m^{\frac{2^{*}}{2} r}}\right)^{2 / 2^{*}} d x-\frac{4}{r^{2}} \int_{\mathbb{T}^{d}} \frac{1}{m^{r}} d x .
$$

By combining Proposition 6 and the above inequalities with the estimate

$$
\int_{\mathbb{T}^{d}} \frac{1}{m^{r}} d x \leq \varepsilon\left(\int_{\mathbb{T}^{d}} \frac{1}{m^{r}} d x\right)^{\beta}+C_{\varepsilon}, \forall \varepsilon>0
$$

we obtain

$$
\int_{\mathbb{T}^{d}} \frac{1}{m^{r}(x, t)} d x \leq C+C \int_{0}^{t}\left(\int_{\mathbb{T}^{d}} \frac{1}{m^{r}} d x\right)^{\beta} d t, \forall t \in[0, T]
$$

Let $h(t)=\int_{\mathbb{T}^{d}} \frac{1}{m(x, t)^{r}} d x$ and $H(t)=\int_{0}^{t} h^{\beta}(s) d s$. Then, the previous inequality reads

$$
h(t) \leq C_{r, \gamma, T}+C_{r, \gamma, T} H(t) .
$$


Thus,

$$
\dot{H}(t)=h^{\beta}(t) \leq C_{r, \alpha, \gamma, T}(1+H(t))^{\beta} .
$$

Integrating (16) and taking into account that $H(0)=1$, we get

$$
(1+H(t))^{1-\beta} \geq 1-(\beta-1) C_{r, \gamma, T} t .
$$

Accordingly,

$$
H(t) \leq \frac{1}{\left[1-(\beta-1) C_{r, \gamma, T} t\right]^{\frac{1}{\beta-1}}}, \text { for all } t<t_{1}(r):=\frac{1}{(\beta-1) C_{r, \gamma, T}} .
$$

Consequently,

$$
\int_{\mathbb{T}^{d}} \frac{1}{m(x, t)^{r}} d x=h(t) \leq C_{r, \gamma, T}+C_{r, \gamma, T} H(t) \leq C+\frac{C}{\left(t_{1}-t\right)^{\frac{1}{\beta-1}}}, t<t_{1} .
$$

Corollary 3. Suppose Assumptions 10.9 hold. Let $r_{0}$ and $t_{1}(r)$ be as in Theorem [. For $r>r_{0}$, let $t \leq t_{1}(r) \equiv t_{1}$. Then, there exists a constant $C_{r}$ and $\delta_{r}$ such that for any $C^{\infty}$ solution $(u, m)$ of (11)

$$
\int_{0}^{t} \int_{\mathbb{T}^{d}}\left|D \frac{1}{m^{r / 2}}\right|^{2} d x d t \leq C_{r}+\frac{C_{r}}{\left(t_{1}-t\right)^{\delta_{r}}}, \quad \forall t<t_{1} .
$$

Iterating the estimates from Proposition [6 we prove next bounds that are uniform in $r$.

Proposition 7. Under Assumptions 1 1, there exist $r_{1}>0$, and constants $C=C(r, \gamma, \alpha)>$ $0, \beta_{r}>1$, such that for any $C^{\infty}$ solution $(u, m)$ to (1) and $r \geq r_{1}$ :

$$
\left\|\frac{1}{m}\right\|_{L^{\infty}\left([0, t] \times \mathbb{T}^{d}\right)} \leq C_{t}\left(1+\left\|\frac{1}{m}\right\|_{L^{\infty}\left([0, t], L^{r}\left(\mathbb{T}^{d}\right)\right)}^{\beta_{r}}\right) .
$$

Proof. For $r>1$, choose $\theta_{n}>0$ such that

$$
r^{n+1}+\delta=\left(1-\theta_{n}\right) r^{n}+\theta_{n} \frac{2^{*}}{2} r^{n+1},
$$

where $\delta=\frac{2 \bar{\alpha}}{2-\gamma}$, that is $\theta_{n}=\frac{1-\frac{1}{r}+\frac{\delta}{r^{n+1}}}{\frac{2^{*}}{2}-\frac{1}{r}}>0$. Set $\lambda_{n}=\frac{2^{*}}{2} \theta_{n}$ and $\beta_{n}=\frac{1-\theta_{n}}{1-\lambda_{n}}$. Then, there exists $r_{1}>1$ such that for any $r \geq r_{1}$ and any $n \geq 1$, we have $\lambda_{n}<1$. We fix a time $t$. As in the previous proposition, using a weighted Holder's inequality, we have

$$
\begin{aligned}
& \int_{\mathbb{T}^{d}} \frac{1}{m^{r^{n+1}+\delta}} d x \leq\left[\left(\int_{\mathbb{T}^{d}} \frac{1}{m^{\frac{2^{*}}{2} r^{n+1}}} d x\right)^{2 / 2^{*}}\right]^{\lambda_{n}}\left[\left(\int_{\mathbb{T}^{d}} \frac{1}{m^{r^{n}}} d x\right)^{\beta_{n}}\right]^{1-\lambda_{n}} \\
& \leq \varepsilon \lambda_{n}\left(\int_{\mathbb{T}^{d}} \frac{1}{m^{\frac{2^{*}}{2} r^{n+1}}} d x\right)^{2 / 2^{*}}+\frac{1}{\varepsilon^{\tau}}\left(1-\lambda_{n}\right)\left(\int_{\mathbb{T}^{d}} \frac{1}{m^{r^{n}}} d x\right)^{\beta_{n}},
\end{aligned}
$$

where $\varepsilon>0$ and $\tau>0$ is a suitable exponent. On the other hand, Proposition 6 and Sobolev's inequality imply

$$
\int_{\mathbb{T}^{d}} \frac{1}{m^{r^{n+1}}(x, t)} d x+\int_{0}^{t}\left(\int_{\mathbb{T}^{d}} \frac{1}{m^{\frac{2^{*}}{2} r^{n+1}(x, s)}} d x\right)^{2 / 2^{*}} d s \leq C_{r^{n+1}}+C_{r^{n+1}} \int_{0}^{t} \int_{\mathbb{T}^{d}} \frac{1}{m^{r^{n+1}+\delta}(x, s)} d x d s .
$$

From these two inequalities, we conclude:

$$
\int_{\mathbb{T}^{d}} \frac{1}{m^{r^{n+1}}(x, t)} d x \leq C_{r^{n+1}}+C_{r^{n+1}} \int_{0}^{t}\left(\int_{\mathbb{T}^{d}} \frac{1}{m^{r^{n}}(x, s)} d x\right)^{\beta_{n}} d s .
$$

Define $A_{n}(t)=\max _{[0, t]} \int_{\mathbb{T}^{d}} \frac{1}{m^{r^{n}}(x, \cdot)} d x$. From the above estimate,

$$
1+A_{n+1}(t) \leq \max \{1, t\} C_{n}\left(1+A_{n}(t)\right)^{\beta_{n}},
$$


where $C_{n}=O\left(r^{n k}\right)$, for some $k>1$. Proceeding inductively, we get

$$
\left(1+A_{n+1}(t)\right)^{\frac{1}{\beta_{1} \cdots \cdot \beta_{n}}} \leq C_{t}^{\sum_{i=1}^{n} \frac{1}{\beta_{1} \cdots \cdot \beta_{i}}} r^{\sum_{i=1}^{n} \frac{i k}{\beta_{1} \cdots \cdot \beta_{i}}}\left(1+A_{1}\right) .
$$

Since

$$
\beta_{n}=\frac{1-\theta_{n}}{1-\lambda_{n}}=r\left(1+\frac{\left(\frac{2^{*}}{2} r-1\right) \delta}{r^{n+1}\left(\frac{2^{*}}{2}-1-\frac{2^{*}}{2} \frac{\delta}{r^{n}}\right)}\right):=r\left(1+q_{n}\right),
$$

where $q_{n}=O\left(r^{-n}\right)>0$, the series $\sum_{i=1}^{\infty} \frac{i k}{\beta_{1} \cdot \ldots \cdot \beta_{i}}, \sum_{i=1}^{\infty} \frac{1}{\beta_{1} \cdot \ldots \cdot \beta_{i}}$, and the infinite product $\prod_{i=1}^{\infty}(1+$ $\left.q_{i}\right)$ converge. From this, we obtain

$$
\left\|\frac{1}{m}\right\|_{L^{\infty}\left([0, t], L^{r n+1}\left(\mathbb{T}^{d}\right)\right)} \leq C_{t}\left(1+\left\|\frac{1}{m}\right\|_{L^{\infty}\left([0, t], L^{r}\left(\mathbb{T}^{d}\right)\right)}^{\beta_{r}}\right),
$$

for some constants $C_{t}>0$ and $\beta_{r}=\prod_{i=1}^{\infty}\left(1+q_{i}\right)>1$ that do not depend on the solution. Sending $n \rightarrow \infty$ yields the result.

The results of Theorem 2] Proposition 7 and Corollary 3 prove the following:

Theorem 3. Under Assumptions 1], there exist a time $T_{0}>0$ and constants $C=C(\gamma, \alpha)>0$, such that for any $C^{\infty}$ solution $(u, m)$ to (1):

$$
\left\|\frac{1}{m}\right\|_{L^{\infty}\left(\left[0, T_{0}\right] \times \mathbb{T}^{d}\right)} \leq C .
$$

\section{Short-time Regularity of the VAlue FUnCtion}

Building upon the results in the previous Section, we prove next further regularity for the solutions of (1).

Lemma 2. Let $w: \mathbb{T}^{d} \times[0, T] \rightarrow \mathbb{R}$ be a non-negative solution of the Fokker-Planck equation:

$$
w_{t}(x, t)-\Delta w-\operatorname{div}(g(x, t) w(x, t))=0,
$$

with $w(x, 0)=m_{0}(x)$. Assume that for some $p_{0}>d$, every $r>1$ and some constants $C_{r}>0$ the drift $g$ satisfies $\|g\|_{L^{r}\left(\left[0, T_{0}\right], L^{\left.p_{0}\left(\mathbb{T}^{d}\right)\right)}\right.} \leq C_{r}$. Then, there exist constants $C_{q}$ such that $\|w\|_{L^{\infty}\left(\left[0, T_{0}\right], L^{q}\left(\mathbb{T}^{d}\right)\right)} \leq$ $C_{q}$, for all $q>1$.

Proof. Multiplying (18) by $q w^{q-1}$ and integrating by parts, we get

$$
\begin{gathered}
\int_{\mathbb{T}^{d}} w^{q}(x, t) d x-\int_{\mathbb{T}^{d}} w^{q}(x, 0) d x+q(q-1) \int_{0}^{t} \int_{\mathbb{T}^{d}} w^{q-2}(x, s)|D w(x, s)|^{2} d x d s \\
=q(q-1) \int_{0}^{t} \int_{\mathbb{T}^{d}} w^{q-1} g \cdot D w d x d s .
\end{gathered}
$$

From this, using Cauchy inequality, we have the estimate

$$
\int_{\mathbb{T}^{d}} w^{q}(x, t) d x+\int_{0}^{t} \int_{\mathbb{T}^{d}}\left|D w^{\frac{q}{2}}(x, s)\right|^{2} d x d s \leq C_{q}+C_{q} \int_{0}^{t} \int_{\mathbb{T}^{d}}|g|^{2} w^{q} d x d s .
$$

The previous bound together with Sobolev's inequality implies

$$
\begin{aligned}
& \int_{0}^{t}\left\|w^{q}(\cdot, s)\right\|_{L^{\frac{2^{*}}{2}\left(\mathbb{T}^{d}\right)}} d s \leq C_{q} \int_{0}^{t} \int_{\mathbb{T}^{d}}\left(1+|g|^{2}\right) w^{q} d x d s \\
& \leq \tilde{C}_{q}\left(1+\int_{0}^{t}\left\|1+|g(\cdot, s)|^{2}\right\|_{L^{p_{1}\left(\mathbb{T}^{d}\right)}}^{r} d s+\int_{0}^{t}\left\|w^{q}(\cdot, s)\right\|_{L^{p^{\prime}\left(\mathbb{T}^{d}\right)}}^{r^{\prime}} d s\right),
\end{aligned}
$$

for any $r>1$, where $p_{1}=\frac{p_{0}}{2}$ and the conjugate powers $r^{\prime}, p_{1}^{\prime}$ satisfy $\frac{1}{r}+\frac{1}{r^{\prime}}=1, \frac{1}{p_{1}}+\frac{1}{p_{1}^{\prime}}=1$. Recall that $\|w(\cdot, t)\|_{L^{1}\left(\mathbb{T}^{d}\right)}=1$. Moreover, because $p_{1}>\frac{d}{2}$, we have $p_{1}^{\prime}<\frac{2^{*}}{2}$. Therefore, by interpolation,

$$
\|w(\cdot, s)\|_{L^{q p_{1}^{\prime}\left(\mathbb{T}^{d}\right)}} \leq\|w(\cdot, s)\|_{L^{q \frac{2^{*}}{2}\left(\mathbb{T}^{d}\right)}}^{\theta}\|w(\cdot, s)\|_{L^{1}\left(\mathbb{T}^{d}\right)}^{1-\theta}=\|w(\cdot, s)\|_{L^{q^{\frac{2}{2}}\left(\mathbb{T}^{d}\right)}}^{\theta},
$$


for some $\theta<1$. Hence, $\left\|w^{q}(\cdot, s)\right\|_{L^{p_{1}^{\prime}\left(\mathbb{T}^{d}\right)}} \leq\left\|w^{q}(\cdot, s)\right\|_{L^{\frac{2^{*}}{2}\left(\mathbb{T}^{d}\right)}}^{\theta}$. By combining these bounds, we have the estimate

$$
\int_{0}^{t}\left\|w^{q}(\cdot, s)\right\|_{L^{\frac{2 *}{2}}\left(\mathbb{T}^{d}\right)} d s \leq C_{q}\left(\int_{0}^{t}\left\|1+|g(\cdot, s)|^{2}\right\|_{L^{p_{1}}\left(\mathbb{T}^{d}\right)}^{r} d s+\int_{0}^{t}\left\|w^{q}(\cdot, s)\right\|_{L^{\frac{2^{*}}{2}}\left(\mathbb{T}^{d}\right)}^{r^{\prime} \theta^{\prime}} d s\right) .
$$

Finally, by choosing $r$ large enough so that $r^{\prime} \theta<1$, we obtain $\int_{0}^{t}\left\|w^{q}(\cdot, s)\right\|_{L^{\frac{2^{*}}{2}\left(\mathbb{T}^{d}\right)}} d s \leq C_{q}$. To end the proof, we observe that, from (19), it follows that, for any $q>1$, there exists $C_{q}$ such that $\int_{\mathbb{T}^{d}} w^{q}(x, t) d x \leq C_{q}$, for any $t \in[0, T]$.

The next Lemma uses the Gagliardo Niremberg theorem to obtain additional regularity. This is a critical point where we use the hypothesis that $H$ is subquadratic.

Lemma 3. Under Assumptions [1], there exist a time $T_{0}>0$ and constants $C_{p}, C_{r, p}=C(\gamma, \alpha, r, p)>$ 0 , such that for any $C^{\infty}$ solution $(u, m)$ to (10):

Furthermore,

$$
\begin{aligned}
\left\|u_{t}\right\|_{L^{r}\left(0, T_{0} ; L^{p}\left(\mathbb{T}^{d}\right)\right)}+\left\|D^{2} u\right\|_{L^{r}\left(0, T_{0} ; L^{p}\left(\mathbb{T}^{d}\right)\right)} & \leq C_{r, p}, \quad \forall 1<r, p<+\infty . \\
\|m\|_{L^{\infty}\left(0, T_{0} ; L^{p}\left(\mathbb{T}^{d}\right)\right)} & \leq C_{p},
\end{aligned}
$$

$$
\|D u\|_{L^{p \gamma\left(\mathbb{T}^{d} \times\left[0, T_{0}\right]\right)}} \leq C, \quad \forall p>1 .
$$

Proof. We choose $T_{0}$ as in Theorem 3 By the Gagliardo-Nirenberg interpolation inequality and Corollary 2 taking into account that $\gamma<2$,

$$
\|D u(\cdot, t)\|_{L^{p \gamma\left(\mathbb{T}^{d}\right)}} \leq c_{p, d}\left\|D^{2} u(\cdot, t)\right\|_{L^{p}\left(\mathbb{T}^{d}\right)}^{\frac{1}{2}}\|u(\cdot, t)\|_{L^{\infty}\left(\mathbb{T}^{d}\right)}^{\frac{1}{2}} \leq C\left\|D^{2} u(\cdot, t)\right\|_{L^{p}\left(\mathbb{T}^{d}\right)}^{\frac{1}{2}} .
$$

For this reason, we have the bound

$$
\begin{aligned}
& \left\||D u|^{\gamma}\right\|_{L^{r}\left(0, T_{0} ; L^{p}\left(\mathbb{T}^{d}\right)\right)}=\left(\int_{0}^{T_{0}}\|D u(\cdot, t)\|_{L^{p \gamma}\left(\mathbb{T}^{d}\right)}^{r \gamma} d t\right)^{\frac{1}{r}} \leq \\
& C\left(\int_{0}^{T_{0}}\left\|D^{2} u(\cdot, t)\right\|_{L^{p}\left(\mathbb{T}^{d}\right)}^{\frac{r \gamma}{2}} d t\right)^{\frac{1}{r}} \leq C\left\|D^{2} u\right\|_{L^{r}\left(0, T_{0} ; L^{p}\left(\mathbb{T}^{d}\right)\right)}^{\frac{\gamma}{2}},
\end{aligned}
$$

where we used again that $\frac{\gamma}{2}<1$. Then, from Theorem 3 and standard regularity results for the heat equation (see, for instance, [27), we have

$$
\begin{aligned}
\left\|u_{t}\right\|_{L^{r}\left(0, T_{0} ; L^{p}\left(\mathbb{T}^{d}\right)\right)}+\left\|D^{2} u\right\|_{L^{r}\left(0, T_{0} ; L^{p}\left(\mathbb{T}^{d}\right)\right)} & \leq C\left\|\left.D u\right|^{\gamma}\right\|_{L^{r}\left(0, T_{0} ; L^{p}\left(\mathbb{T}^{d}\right)\right)}+C\left\|m^{\alpha}\right\|_{L^{r}\left(0, T_{0} ; L^{p}\left(\mathbb{T}^{d}\right)\right)} \\
& \leq C\left\|D^{2} u\right\|_{L^{r}\left(0, T_{0} ; L^{p}\left(\mathbb{T}^{d}\right)\right)}^{\frac{\gamma}{2}}+C\left\|m^{\alpha}\right\|_{L^{r}\left(0, T_{0} ; L^{p}\left(\mathbb{T}^{d}\right)\right)} .
\end{aligned}
$$

Since $\frac{\gamma}{2}<1$, we obtain

$$
\left\|u_{t}\right\|_{L^{r}\left(0, T_{0} ; L^{p}\left(\mathbb{T}^{d}\right)\right)} C\left\|D^{2} u\right\|_{L^{r}\left(0, T_{0} ; L^{p}\left(\mathbb{T}^{d}\right)\right)} \leq C\left\|m^{\alpha}\right\|_{L^{r}\left(0, T_{0} ; L^{p}\left(\mathbb{T}^{d}\right)\right)} .
$$

The above arguments also imply

$$
\|D u\|_{L^{r \gamma}\left(0, T_{0} ; L^{p \gamma}\left(\mathbb{T}^{d}\right)\right)} \leq C\left\|D^{2} u\right\|_{L^{r}\left(0, T_{0} ; L^{p}\left(\mathbb{T}^{d}\right)\right)}^{\frac{1}{2}} \leq C+C\left\|m^{\alpha}\right\|_{L^{r}\left(0, T_{0} ; L^{p}\left(\mathbb{T}^{d}\right)\right)}^{\frac{1}{2}} \leq C_{r, p},
$$

for all $r, p>1$.

We recall that $\alpha<\frac{d-2}{d}$. By Proposition [4, we have $\left\|m^{\alpha+1}\right\|_{L^{\infty}\left(0, T_{0} ; L^{1}\left(\mathbb{T}^{d}\right)\right)} \leq C$. Hence, for some $p_{0}>\frac{d}{2},\left\|m^{\alpha}\right\|_{L^{\infty}\left(0, T_{0} ; L^{p_{0}}\left(\mathbb{T}^{d}\right)\right)} \leq C$. Consequently, $\|D u\|_{L^{r}\left(0, T_{0} ; L^{p_{0} \gamma}\left(\mathbb{T}^{d}\right)\right)} \leq C_{r}$, for any $r>1$. Note that $p_{0} \gamma /(\gamma-1)>\frac{d}{2} \gamma /(\gamma-1)>d$. Therefore, $D_{p} H$ is bounded in $L^{r}\left(0, T_{0} ; L^{p}\left(\mathbb{T}^{d}\right)\right)$ for some $p>d$ and any $r>1$. Finally, Lemma 2 implies that $m$ is bounded in $L^{\infty}\left(0, T_{0} ; L^{q}\left(\mathbb{T}^{d}\right)\right)$ for any $q>1$.

Lemma 4. Under Assumptions [1], there exist a time $T_{0}>0$ and a constant $C>0$, such that,

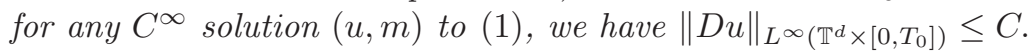


Proof. From Theorem 3 and Lemma 3 it follows that the equation for $u$ can be written as

$$
\left\{\begin{array}{l}
u_{t}+\Delta u=f \\
u\left(x, T_{0}\right)=\Psi(x)
\end{array}\right.
$$

where $f \in L^{r}\left(\mathbb{T}^{d} \times\left[0, T_{0}\right]\right)$ for every $r>1$. From this, reasoning as in [16], we obtain

$$
\|D u\|_{L^{\infty}\left(\mathbb{T}^{d} \times\left[0, T_{0}\right]\right)} \leq C .
$$

Lemma 5. Under Assumptions [1], there exist a time $T_{0}>0$ and a constant $C>0$ such that, for any $C^{\infty}$ solution $(u, m)$ to (10), we have $\|m\|_{L^{\infty}\left(\mathbb{T}^{d} \times\left[0, T_{0}\right]\right)},\|D m\|_{L^{\infty}\left(\mathbb{T}^{d} \times\left[0, T_{0}\right]\right)} \leq C$.

Proof. From the estimates in Theorem 3 and Lemmas 3 and 4 it follows that for suitable functions $a$, and $c$, bounded in $L^{p}\left(\mathbb{T}^{d} \times\left[0, T_{0}\right]\right)$ for every $p>1$, the equation for $m$ can be written as

$$
\left\{\begin{array}{l}
-m_{t}(x, t)+\Delta m(x, t)=a(x, t) \cdot D m(x, t)+c(x, t) m(x, t), \\
m(x, 0)=m_{0}(x) .
\end{array}\right.
$$

Let $w=\ln m$ then

$$
\left\{\begin{array}{l}
-w_{t}(x, t)+\Delta w(x, t)+|D w|^{2}-a(x, t) \cdot D w(x, t)=c(x, t), \\
w(x, 0)=\ln m_{0}(x) .
\end{array}\right.
$$

The adjoint method, applied as in [12, yields $\|w\|_{L^{\infty}\left(\mathbb{T}^{d} \times\left[0, T_{0}\right]\right)},\|D w\|_{L^{\infty}\left(\mathbb{T}^{d} \times\left[0, T_{0}\right]\right)} \leq C$. These estimates imply the result.

Theorem 4. Under Assumptions 1, 2, there exist a time $T_{0}>0$ and constants $C_{k, l, p}>0$, for $k, l \in \mathbb{N}, p>1$ such that, for any $C^{\infty}$ solution $(u, m)$ to (1), we have $\left\|D_{t}^{k} D_{x}^{l} u\right\|_{L^{p}\left(\mathbb{T}^{d} \times\left[0, T_{0}\right]\right)} \leq$ $C_{k, l, p}$.

Proof. The result follows by a simple bootstrapping argument. As a starting point, we use the regularity given by Theorem 3 and Lemmas 3 , 4 , and 5 . Then, the the Theorem is proven by repeatedly using the parabolic regularity on the equations for $u, m$, and their derivatives.

\section{Existence of solutions}

To establish the existence of solutions, we will use the continuation method. For that, we introduce the problem

$$
\left\{\begin{array}{l}
-u_{t}-\Delta u+m^{\alpha} H_{\lambda}\left(x, \frac{D u}{m^{\alpha}}\right)+b_{\lambda} \cdot D u=V_{\lambda}(x, m(x, t)), \\
m_{t}-\Delta m-\operatorname{div}\left(D_{p} H_{\lambda}\left(x, \frac{D u}{m^{\alpha}}\right) m\right)-\operatorname{div}\left(b_{\lambda} m\right)=0, \\
u(x, T)=\Psi_{\lambda}(x), m(x, 0)=m_{\lambda}(x),
\end{array}\right.
$$

where $0 \leq \lambda \leq 1, H_{\lambda}(x, p)=(1-\lambda) H_{0}+\lambda\left(1+|p|^{2}\right)^{\frac{\gamma}{2}}, b_{\lambda}=(1-\lambda) b, V_{\lambda}=(1-\lambda) V+\lambda \arctan (m)$, $\Psi_{\lambda}=(1-\lambda) \Psi, m_{\lambda}=(1-\lambda) m_{0}+\lambda$. The terminal time $T$ satisfies $T \in \mathcal{T}=\left[0, T_{0}\right]$, where $T_{0}$ is as in Theorem 4 .

When $\lambda=1$, 200 has a unique solution, namely $u \equiv\left(1-\frac{\pi}{4}\right) t, m \equiv 1$. We will prove that the set $\Lambda$ of values $0 \leq \lambda \leq 1$ for which (20) admits a solution is relatively open and closed. Therefore, $\Lambda=[0,1]$ and, in particular, (1) admits a solution.

For $k \geq-1$, we set $F^{k}\left(\mathcal{T} ; \mathbb{T}^{d}\right)=\cap_{2 k_{1}+k_{2}=k} H^{k_{1}}\left(\mathcal{T} ; H^{k_{2}}\left(\mathbb{T}^{d}\right)\right)$, where the intersection is taken over all integers $k_{1} \geq 0, k_{2} \geq-1$. The space $F^{k}\left(\mathcal{T} ; \mathbb{T}^{d}\right)$ is a Banach space endowed with the norm

$$
\|f\|_{F^{k}\left(\mathcal{T} ; \mathbb{T}^{d}\right)}=\sum_{2 k_{1}+k_{2}=k}\|f\|_{H^{k_{1}}\left(\mathcal{T} ; H^{\left.k_{2}\left(\mathbb{T}^{d}\right)\right)}\right.} .
$$

Moreover, there exists $\tilde{k}_{d}$, depending only on the dimension $d$, such that for $k \geq \tilde{k}$, the space $F^{k-2}$ is an algebra. Let $k \geq \tilde{k}_{d}$, and consider the operator

$$
\mathcal{M}_{\lambda}: F^{k}\left(\mathcal{T} ; \mathbb{T}^{d}\right) \times F^{k}\left(\mathcal{T} ; \mathbb{T}^{d}\right) \rightarrow F^{k-2}\left(\mathcal{T} ; \mathbb{T}^{d}\right) \times F^{k-2}\left(\mathcal{T} ; \mathbb{T}^{d}\right) \times H^{k-1}\left(\mathbb{T}^{d}\right) \times H^{k-1}\left(\mathbb{T}^{d}\right)
$$


given by

$$
\mathcal{M}_{\lambda}\left[\begin{array}{c}
u \\
m
\end{array}\right]=\left[\begin{array}{c}
m_{t}-\Delta m-\operatorname{div}\left(D_{p} H_{\lambda}\left(x, \frac{D u}{m^{\alpha}}\right) m\right)+\operatorname{div}\left(b_{\lambda} m\right) \\
u_{t}+\Delta u-m^{\alpha} H_{\lambda}\left(x, \frac{D u}{m^{\alpha}}\right)-b_{\lambda} \cdot D u+V_{\lambda}(x, m) \\
m(x, 0)-m_{\lambda}(x) \\
u(x, T)-\Psi_{\lambda}(x)
\end{array}\right] .
$$

Then, (20) is equivalent to

$$
\mathcal{M}_{\lambda}\left[\begin{array}{c}
u \\
m
\end{array}\right]=0
$$

and (11) then reads as $\mathcal{M}_{0}\left[\begin{array}{c}u \\ m\end{array}\right]=0$. Moreover, as we remarked before, $\mathcal{M}_{1}\left[\begin{array}{c}u \\ m\end{array}\right]=0$, has only the trivial solution $u \equiv\left(1-\frac{\pi}{4}\right) t, m \equiv 1$. We consider the linearized operator $\mathcal{L}$ :

$$
\mathcal{L}_{\lambda}\left[\begin{array}{l}
v \\
f
\end{array}\right]=\lim _{\varepsilon \rightarrow 0} \frac{\mathcal{M}_{\lambda}\left[\begin{array}{c}
u+\varepsilon v \\
m+\varepsilon f
\end{array}\right]-\mathcal{M}_{\lambda}\left[\begin{array}{c}
u \\
m
\end{array}\right]}{\varepsilon}=
$$

$$
\left[\begin{array}{c}
f_{t}-\Delta f-\operatorname{div}\left[D_{p} H_{\lambda}\left(x, \frac{D u}{m^{\alpha}}\right) f+m^{1-\alpha} D_{p p}^{2} H_{\lambda}\left(x, \frac{D u}{m^{\alpha}}\right) \cdot D v-\alpha f D_{p p}^{2} H_{\lambda}\left(x, \frac{D u}{m^{\alpha}}\right) \cdot \frac{D u}{m^{\alpha}}+b_{\lambda} f\right] \\
v_{t}+\Delta v-\alpha m^{\alpha-1} f\left(H_{\lambda}\left(x, \frac{D u}{m^{\alpha}}\right)-\frac{D u}{m^{\alpha}} D_{p} H_{\lambda}\left(x, \frac{D u}{m^{\alpha}}\right)\right)-D_{p} H_{\lambda}\left(x, \frac{D u}{m^{\alpha}}\right) D v-b_{\lambda} \cdot D v+D_{z} V_{\lambda} f \\
f(x, 0) \\
v(x, T)
\end{array}\right]
$$

Note that $\mathcal{L}_{\lambda}: F^{k}\left(\mathcal{T} ; \mathbb{T}^{d}\right) \times F^{k}\left(\mathcal{T} ; \mathbb{T}^{d}\right) \rightarrow F^{k-2}\left(\mathcal{T} ; \mathbb{T}^{d}\right) \times F^{k-2}\left(\mathcal{T} ; \mathbb{T}^{d}\right) \times H^{k-1}\left(\mathbb{T}^{d}\right) \times H^{k-1}\left(\mathbb{T}^{d}\right)$, for all $k$ large enough. However, if $u$ and $m$ are $C^{\infty}$ solutions to (20), then $\mathcal{L}_{\lambda}$ admits a unique extension as bounded linear operator $\mathcal{L}_{\lambda}: F^{k}\left(\mathcal{T} ; \mathbb{T}^{d}\right) \times F^{k}\left(\mathcal{T} ; \mathbb{T}^{d}\right) \rightarrow F^{k-2}\left(\mathcal{T} ; \mathbb{T}^{d}\right) \times F^{k-2}\left(\mathcal{T} ; \mathbb{T}^{d}\right) \times$ $H^{k-1}\left(\mathbb{T}^{d}\right) \times H^{k-1}\left(\mathbb{T}^{d}\right)$, for all $k \geq 1$.

The form $\langle\cdot, \cdot\rangle$ denotes the scalar product on $L^{2}\left(\mathbb{T}^{d}\right)$. To apply the inverse function theorem, we need to prove that the linear operator $\mathcal{L}_{\lambda}$ is invertible. For this, we begin by showing that the equation $\mathcal{L}_{\lambda} w=W$ has a unique weak solution in the sense of the following definition:

Definition 1. For $h, g \in L^{2}\left(0, T_{0} ; L^{2}\left(\mathbb{T}^{d}\right)\right), A, B \in L^{2}\left(\mathbb{T}^{d}\right)$, set

$$
W(x, t)=\left[\begin{array}{c}
h(x, t) \\
g(x, t) \\
A(x) \\
B(x)
\end{array}\right]
$$

A function $w=\left[\begin{array}{l}v \\ f\end{array}\right]$, with

(22) $\quad v, f \in L^{2}\left(0, T_{0} ; H^{1}\left(\mathbb{T}^{d}\right)\right)$ and $v_{t}, f_{t} \in L^{2}\left(0, T_{0} ; H^{-1}\left(\mathbb{T}^{d}\right)\right)$, that is $v, f \in F^{1}\left(\mathcal{T} ; \mathbb{T}^{d}\right)$,

is a weak solution of $\mathcal{L}_{\lambda} w=W$ if:

1. for any $\bar{v}, \bar{f} \in H^{1}\left(\mathbb{T}^{d}\right)$ and for a.e. $t, 0 \leq t \leq T_{0}$ we have

$$
\left\{\begin{array}{l}
\left\langle f_{t}, \bar{f}\right\rangle+\left\langle D f+D_{p} H_{\lambda} f+m^{1-\alpha} D_{p p}^{2} H_{\lambda} \cdot D v-\alpha f D_{p p}^{2} H_{\lambda} \cdot Q+f b_{\lambda}, D \bar{f}\right\rangle=\langle h, \bar{f}\rangle \\
\left\langle v_{t}, \bar{v}\right\rangle-\langle D v, D \bar{v}\rangle-\left\langle\alpha m^{\alpha-1} f\left(H_{\lambda}-Q \cdot D_{p} H_{\lambda}\right)+D_{p} H_{\lambda} \cdot D v+b_{\lambda} \cdot D v-D_{z} V_{\lambda} f, \bar{v}\right\rangle=\langle g, \bar{v}\rangle,
\end{array}\right.
$$

here $Q=\frac{D u}{m^{\alpha}}$ and the Hamiltonian $H_{\lambda}$ and its derivative are evaluated at the point $(x, Q)$.

2. $f(x, 0)=A(x), v\left(x, T_{0}\right)=B(x)$. 
Remark 6. Note that (22) implies v, $f \in C\left(\mathcal{T} ; L^{2}\left(\mathbb{T}^{d}\right)\right)$ ( see e.g. [4], Section 5.9.2, Theorem 3). Therefore, the traces $f(x, 0), v\left(x, T_{0}\right)$ are well-defined.

Theorem 5 (Uniqueness of weak solutions). Let $\left(u_{\lambda}, m_{\lambda}\right)$ be a $C^{\infty}$ solution to (20), and let $T_{0}$ be as in Theorem 4. Then, under Assumptions 11 12, there exists at most one weak solution to the equation $\mathcal{L}_{\lambda} w=W$ in the sense of Definition 1 .

Proof. Since the equation $\mathcal{L}_{\lambda} w=W$ is linear, it is enough to prove that $\mathcal{L}_{\lambda} w=0$ has only the trivial solution $w=0$. For this, we take $\bar{f}=v, \bar{v}=f$ in (23). Adding both equations and integrating in time, we obtain

$$
\begin{aligned}
0= & \int_{0}^{T_{0}} \int_{\mathbb{T}^{d}}\left[\alpha m^{\alpha-1} f^{2}\left(Q \cdot D_{p} H_{\lambda}-H_{\lambda}\right)+m^{1-\alpha} D v \cdot D_{p p}^{2} H_{\lambda} \cdot D v\right. \\
& \left.-\alpha f Q \cdot D_{p p}^{2} H_{\lambda} \cdot D v+D_{z} V_{\lambda} f^{2}\right] d x d t= \\
& \int_{0}^{T_{0}} \int_{\mathbb{T}^{d}}\left[\alpha m^{\alpha-1} f^{2}\left(Q \cdot D_{p} H_{\lambda}-H_{\lambda}-\frac{\alpha}{4} Q \cdot D_{p p}^{2} H_{\lambda} \cdot Q\right)\right. \\
& \left.+m^{\alpha-1}\left(m^{1-\alpha} D v-\frac{\alpha}{2} f Q\right)^{t} \cdot D_{p p}^{2} H_{\lambda} \cdot\left(m^{1-\alpha} D v-\frac{\alpha}{2} f Q\right)+D_{z} V_{\lambda} f^{2}\right] d x d t,
\end{aligned}
$$

where we set $Q=\frac{D u}{m^{\alpha}}$. Using the estimates from Theorem 3 , Lemma 4 Remark 4 , and Assumption 12. we conclude that at a solution $\left(u_{\lambda}, m_{\lambda}\right)$ to (20) there exist constants $\theta_{1}, \theta_{2}>0$ that do not depend on the solution and $\lambda$, such that the above expression bounded by below by

$$
\theta_{1} \int_{0}^{T_{0}} \int_{\mathbb{T}^{d}} m^{\alpha-1}\left|m^{1-\alpha} D v-\frac{\alpha}{2} f Q\right|^{2}+\theta_{2}|f|^{2} d x d t .
$$

Thus, we get $f=0, D v=0$. Consequently $v \equiv v(t)$. Next, by looking at the second equation in (23), for $\bar{v}=v(t)$ and $\bar{f}=0$, we obtain

$$
\frac{d}{d t}\langle v, v\rangle=0
$$

Using the boundary conditions for $v$, we conclude that $v=0$. Therefore, $w=0$.

To prove the existence of weak solutions, we apply the Galerkin approximation method (see e.g. [4]). We consider a sequence of $C^{\infty}$ functions $e_{k}=e_{k}(x), k \in \mathbb{N}$ such that $\left\{e_{k}\right\}_{k=1}^{\infty}$ is an orthogonal basis of $H^{1}\left(\mathbb{T}^{d}\right)$ and an orthonormal basis of $L^{2}\left(\mathbb{T}^{d}\right)$. We construct a sequence of finite dimensional approximations to weak solutions of (20) as follows, let $v_{N}, f_{N}:\left[0, T_{0}\right] \rightarrow H^{1}\left(\mathbb{T}^{d}\right)$

$$
f_{N}(t)=\sum_{k=1}^{N} A_{N}^{k}(t) e_{k}, \quad v_{N}(t)=\sum_{k=1}^{N} B_{N}^{k}(t) e_{k} .
$$

We will show that we can select the coefficients $A_{n}^{k}, B_{N}^{k}$ so that

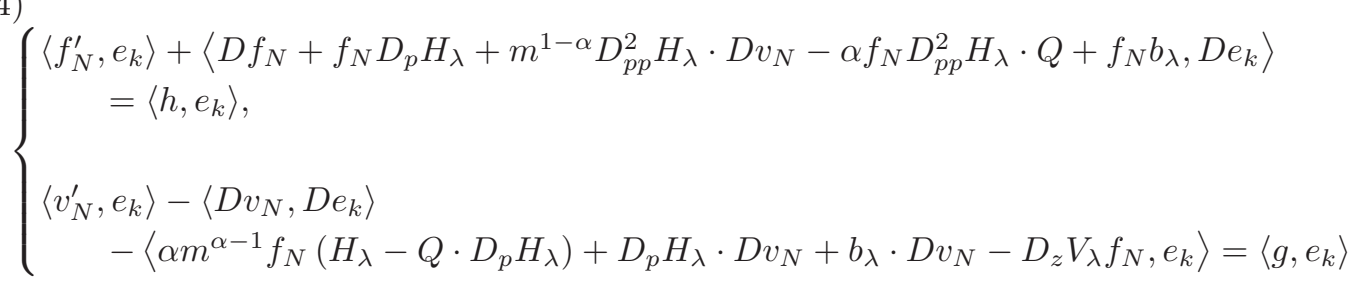

and

$$
A_{N}^{k}(0)=\left\langle A, e_{k}\right\rangle, B_{N}^{k}\left(T_{0}\right)=\left\langle B, e_{k}\right\rangle, \quad k=1,2, \ldots, N
$$


The system (24) is equivalent to:

$$
\left\{\begin{aligned}
\dot{A}_{N}^{k} & +\sum_{l=1}^{N}\left\langle D e_{l}+e_{l} D_{p} H_{\lambda}-\alpha e_{l} D_{p p}^{2} H_{\lambda} \cdot Q+e_{l} b_{\lambda}, D e_{k}\right\rangle A_{N}^{l} \\
& +\sum_{l=1}^{N}\left\langle m^{1-\alpha} D_{p p}^{2} H_{\lambda} \cdot D e_{l}, D e_{k}\right\rangle B_{N}^{l}=\left\langle h, e_{k}\right\rangle, \\
\dot{B}_{N}^{k} & -\sum_{l=1}^{N}\left\langle D e_{l}+D_{p} H_{\lambda} \cdot D e_{l}+b_{\lambda} \cdot D e_{l}, D e_{k}\right\rangle B_{N}^{l} \\
& \quad-\sum_{l=1}^{N}\left\langle\alpha m^{\alpha-1} e_{l}\left(H_{\lambda}-Q \cdot D_{p} H_{\lambda}\right)-e_{l} D_{z} V_{\lambda}, e_{k}\right\rangle A_{N}^{l}=\left\langle g, e_{k}\right\rangle .
\end{aligned}\right.
$$

Because (26) is a linear system of ordinary differential equations, the only difficulty in proving the existence of solutions concerns the boundary conditions (25). Existence is not immediate because half of the boundary conditions are given at the initial time, whereas the other half are given at the terminal time. From standard theory of ordinary differential equations, the initial value problem for (26), that is, with $A_{N}^{k}(0)$ and $B_{N}^{k}(0)$ prescribed, has a unique solution. Hence, to prove the existence of solutions to (26), it is enough to show the existence of solutions for the corresponding homogeneous problem:

$$
\left\{\begin{aligned}
\dot{\tilde{A}}_{n}^{k}+ & \sum_{l=1}^{N}\left\langle D e_{l}+e_{l} D_{p} H_{\lambda}-\alpha e_{l} D_{p p}^{2} H_{\lambda} \cdot Q+e_{l} b_{\lambda}, D e_{k}\right\rangle \tilde{A}_{N}^{l} \\
& +\sum_{l=1}^{N}\left\langle m^{1-\alpha} D_{p p}^{2} H_{\lambda} \cdot D e_{l}, D e_{k}\right\rangle \tilde{B}_{N}^{l}=0 \\
\dot{\tilde{B}}_{N}^{k} & -\sum_{l=1}^{N}\left\langle D e_{l}+D_{p} H_{\lambda} \cdot D e_{l}+b_{\lambda} \cdot D e_{l}, D e_{k}\right\rangle \tilde{B}_{N}^{l} \\
& \quad-\sum_{l=1}^{N}\left\langle\alpha m^{\alpha-1} e_{l}\left(H_{\lambda}-Q \cdot D_{p} H_{\lambda}\right)-e_{l} D_{z} V_{\lambda}, e_{k}\right\rangle \tilde{A}_{N}^{l}=0
\end{aligned}\right.
$$

with arbitrary $\tilde{A}_{N}^{k}(0)$ and $\tilde{B}_{N}^{k}\left(T_{0}\right), 1 \leq k \leq N$. Indeed, any solution to (26)-(25), $(A, B)$ can be written as a sum of a particular solution to (26),$(\bar{A}, \bar{B})$, for instance with

$$
\bar{A}_{N}^{k}(0)=0, \bar{B}_{N}^{k}(0)=0, k=1,2, \ldots, N .
$$

with a solution, $(\tilde{A}, \tilde{B})$ to (27) with suitable initial and terminal conditions so that (25) holds for $(A, B)=(\bar{A}+\tilde{A}, \bar{B}+\tilde{B})$.

Next, we regard the solution of the initial value problem for the homogeneous system corresponding to (26) as a linear operator on $\mathbb{R}^{2 N}$ :

$$
\left(A_{N}(0), B_{N}(0)\right) \mapsto\left(A_{N}(0), B_{N}\left(T_{0}\right)\right) .
$$

We need to prove that this mapping is surjective. Since (28) is a linear mapping from $\mathbb{R}^{2 N}$ to $\mathbb{R}^{2 N}$, surjectivity is equivalent to injectivity. Therefore, it suffices to prove that the homogeneous system of ODE's corresponding to (26) subject to initial-terminal conditions $A_{N}(0)=B_{N}\left(T_{0}\right)=0$ has only the trivial solution $A_{N}=B_{N} \equiv 0$. Let $f_{N}, v_{N}$ solve (24) with $h=g \equiv 0, A=B \equiv 0$. From (24), we obtain (23) for $f=\bar{v}=f_{N}, v=\bar{f}=v_{N}$. Using the same argument as in Theorem 5. we conclude that $f_{N}=v_{N} \equiv 0$.

Next, we prove energy estimates for these approximations to ensure the weak convergence of approximate solutions through some subsequence.

Theorem 6. Suppose Assumptions 112 hold. Then, for $T_{0}$ small enough, there exists a constant $C$ such that for any $C^{\infty}$ solution $\left(u_{\lambda}, m_{\lambda}\right)$ to (20), we have

$$
\begin{gathered}
\max _{0 \leq t \leq T_{0}}\left\|\left(f_{N}, v_{N}\right)\right\|_{\left(L^{2}\left(\mathbb{T}^{d}\right)\right)^{2}}+\left\|\left(f_{N}, v_{N}\right)\right\|_{\left(L^{2}\left(0, T_{0} ; H^{1}\left(\mathbb{T}^{d}\right)\right)\right)^{2}}+\left\|\left(f_{N}^{\prime}, v_{N}^{\prime}\right)\right\|_{\left(L^{2}\left(0, T_{0} ; H^{-1}\left(\mathbb{T}^{d}\right)\right)\right)^{2}} \\
\leq C\left(\|h\|_{L^{2}\left(0, T_{0} ; L^{2}\left(\mathbb{T}^{d}\right)\right)}+\|g\|_{L^{2}\left(0, T_{0} ; L^{2}\left(\mathbb{T}^{d}\right)\right)}+\|A\|_{L^{2}\left(\mathbb{T}^{d}\right)}+\|B\|_{L^{2}\left(\mathbb{T}^{d}\right)}\right) .
\end{gathered}
$$

Proof. We assume $T_{0}$ is small enough so that Theorem 4 holds. Using the linearity of (24), we observe that (23) holds for $f=\bar{f}=f_{N}, v_{N}=\bar{v}_{N}=v_{N}$. Then, using Hölder's inequality and the estimates from Theorem 4, we obtain the system of inequalities:

$$
\left\{\begin{array}{l}
\left(\left\|f_{N}\right\|_{L^{2}\left(\mathbb{T}^{d}\right)}^{2}\right)_{t}+\left\|D f_{N}\right\|_{L^{2}\left(\mathbb{T}^{d}\right)}^{2} \leq C\left(\|h\|_{L^{2}\left(\mathbb{T}^{d}\right)}^{2}+\left\|D v_{N}\right\|_{L^{2}\left(\mathbb{T}^{d}\right)}^{2}+\left\|f_{N}\right\|_{L^{2}\left(\mathbb{T}^{d}\right)}^{2}\right), \\
\left(\left\|v_{N}\right\|_{L^{2}\left(\mathbb{T}^{d}\right)}^{2}\right)_{t}-\left\|D v_{N}\right\|_{L^{2}\left(\mathbb{T}^{d}\right)}^{2} \geq-C\left(\|g\|_{L^{2}\left(\mathbb{T}^{d}\right)}^{2}+\left\|v_{N}\right\|_{L^{2}\left(\mathbb{T}^{d}\right)}^{2}+\left\|f_{N}\right\|_{L^{2}\left(\mathbb{T}^{d}\right)}^{2}\right) .
\end{array}\right.
$$


From the second inequality, using Gronwall's inequality, we get

$$
\left\|v_{N}(\cdot, t)\right\|_{L^{2}\left(\mathbb{T}^{d}\right)}^{2} \leq C \int_{t}^{T_{0}}\left(\|g(\cdot, s)\|_{L^{2}\left(\mathbb{T}^{d}\right)}^{2}+\left\|f_{N}(\cdot, s)\right\|_{L^{2}\left(\mathbb{T}^{d}\right)}^{2}\right) d s+C\|B\|_{L^{2}\left(\mathbb{T}^{d}\right)}^{2},
$$

and further

$$
\int_{0}^{T_{0}}\left\|D v_{N}(\cdot, s)\right\|_{L^{2}\left(\mathbb{T}^{d}\right)}^{2} d s \leq C \int_{0}^{T_{0}}\left(\|g(\cdot, s)\|_{L^{2}\left(\mathbb{T}^{d}\right)}^{2}+\left\|f_{N}(\cdot, s)\right\|_{L^{2}\left(\mathbb{T}^{d}\right)}^{2}\right) d s+C\|B\|_{L^{2}\left(\mathbb{T}^{d}\right)}^{2} .
$$

From this, combined with the first inequality in (29), and using Gronwall's inequality once more, we have

$\left\|f_{N}(\cdot, t)\right\|_{L^{2}\left(\mathbb{T}^{d}\right)}^{2} \leq C \int_{0}^{T_{0}}\left(\|g(\cdot, s)\|_{L^{2}\left(\mathbb{T}^{d}\right)}^{2}+\|h(\cdot, s)\|_{L^{2}\left(\mathbb{T}^{d}\right)}^{2}+\left\|f_{N}(\cdot, s)\right\|_{L^{2}\left(\mathbb{T}^{d}\right)}^{2}\right) d s+C\left(\|A\|_{L^{2}\left(\mathbb{T}^{d}\right)}^{2}+\|B\|_{L^{2}\left(\mathbb{T}^{d}\right)}^{2}\right)$.

Thus, for $T_{0}$ small enough, we get

$\sup _{t \in\left[0, T_{0}\right]}\left\|f_{N}(\cdot, t)\right\|_{L^{2}\left(\mathbb{T}^{d}\right)}^{2} \leq C \int_{0}^{T_{0}}\left(\|g(\cdot, s)\|_{L^{2}\left(\mathbb{T}^{d}\right)}^{2}+\|h(\cdot, s)\|_{L^{2}\left(\mathbb{T}^{d}\right)}^{2}\right) d s+C\left(\|A\|_{L^{2}\left(\mathbb{T}^{d}\right)}^{2}+\|B\|_{L^{2}\left(\mathbb{T}^{d}\right)}^{2}\right)$.

Consequently,

$\int_{0}^{T_{0}}\left\|D f_{N}(\cdot, t)\right\|_{L^{2}\left(\mathbb{T}^{d}\right)}^{2} \leq C \int_{0}^{T_{0}}\left(\|g(\cdot, s)\|_{L^{2}\left(\mathbb{T}^{d}\right)}^{2}+\|h(\cdot, s)\|_{L^{2}\left(\mathbb{T}^{d}\right)}^{2}\right) d s+C\left(\|A\|_{L^{2}\left(\mathbb{T}^{d}\right)}^{2}+\|B\|_{L^{2}\left(\mathbb{T}^{d}\right)}^{2}\right)$.

Thus, we have

$$
\begin{aligned}
& \max _{0 \leq t \leq T_{0}}\left\|\left(f_{N}, v_{N}\right)\right\|_{\left(L^{2}\left(\mathbb{T}^{d}\right)\right)^{2}}+\left\|\left(f_{N}, v_{N}\right)\right\|_{\left(L^{2}\left(0, T_{0} ; H^{1}\left(\mathbb{T}^{d}\right)\right)\right)} \\
& \leq C\left(\|h\|_{L^{2}\left(0, T_{0} ; L^{2}\left(\mathbb{T}^{d}\right)\right)}+\|g\|_{L^{2}\left(0, T_{0} ; L^{2}\left(\mathbb{T}^{d}\right)\right)}+\|A\|_{L^{2}\left(\mathbb{T}^{d}\right)}+\|B\|_{L^{2}\left(\mathbb{T}^{d}\right)}\right) .
\end{aligned}
$$

From equation (23), for any $\bar{f}, \bar{v} \in \operatorname{span}\left\{e_{k}\right\}_{k=1}^{N}$ with $\|\bar{f}\|_{L^{2}\left(0, T_{0} ; H^{1}\left(\mathbb{T}^{d}\right)\right)} \leq 1,\|\bar{v}\|_{L^{2}\left(0, T_{0} ; H^{1}\left(\mathbb{T}^{d}\right)\right)} \leq 1$ we get

$$
\left\{\begin{array}{l}
\int_{0}^{T_{0}}\left\langle f_{N}(s), \bar{f}\right\rangle d s \leq C\left(\|h\|_{L^{2}\left(0, T_{0} ; L^{2}\left(\mathbb{T}^{d}\right)\right)}^{2}+\|g\|_{L^{2}\left(0, T_{0} ; L^{2}\left(\mathbb{T}^{d}\right)\right)}^{2}+\|A\|_{L^{2}\left(\mathbb{T}^{d}\right)}^{2}+\|B\|_{L^{2}\left(\mathbb{T}^{d}\right)}^{2}\right) \\
\int_{0}^{T_{0}}\left\langle v_{N}(s), \bar{v}\right\rangle d s \leq C\left(\|h\|_{L^{2}\left(0, T_{0} ; L^{2}\left(\mathbb{T}^{d}\right)\right)}^{2}+\|g\|_{L^{2}\left(0, T_{0} ; L^{2}\left(\mathbb{T}^{d}\right)\right)}^{2}+\|A\|_{L^{2}\left(\mathbb{T}^{d}\right)}^{2}+\|B\|_{L^{2}\left(\mathbb{T}^{d}\right)}^{2}\right)
\end{array}\right.
$$

since $f_{N}, v_{N} \in \operatorname{span}\left\{e_{k}\right\}_{k=1}^{N}$ as well. These inequalities imply the required estimates.

Theorem 7 (Existence of weak solutions). Let $\left(u_{\lambda}, m_{\lambda}\right)$ be a $C^{\infty}$ solution to (20) and let $T_{0}$ be as in Theorem 4. Then, under Assumptions 11 12, there exists a weak solution to the equation $\mathcal{L}_{\lambda} w=W$ in the sense of (23).

Proof. According to the energy estimates, there exist subsequences of $v_{N}, f_{N}$ and functions $v, f \in$ $L^{2}\left(0, T_{0} ; H^{1}\left(\mathbb{T}^{d}\right)\right)$, with $v^{\prime}=v_{t}, f^{\prime}=f_{t} \in L^{2}\left(0, T_{0} ; H^{-1}\left(\mathbb{T}^{d}\right)\right)$, such that

$$
\left\{\begin{array}{l}
v_{N} \rightarrow v, f_{N} \rightarrow f, \text { weakly in } L^{2}\left(0, T_{0} ; H^{1}\left(\mathbb{T}^{d}\right)\right) \\
v_{N}^{\prime} \rightarrow v^{\prime}, f_{N}^{\prime} \rightarrow f^{\prime}, \text { weakly in } L^{2}\left(0, T_{0} ; H^{-1}\left(\mathbb{T}^{d}\right)\right) .
\end{array}\right.
$$

For fixed $N_{0}$, let $\bar{v}, \bar{f} \in \operatorname{span}\left\{e_{k}\right\}_{k=1}^{N_{0}}$ with $\|\bar{v}\|_{L^{2}\left(0, T_{0} ; H^{1}\left(\mathbb{T}^{d}\right)\right)},\|\bar{f}\|_{L^{2}\left(0, T_{0} ; H^{1}\left(\mathbb{T}^{d}\right)\right)} \leq 1$. According to the definition of $v_{N}, f_{N}$, we have that (23) holds for every $N \geq N_{0}$. Weak convergence then implies (23) for $v, f$ and any $\bar{v}, \bar{f} \in \operatorname{span}\left\{e_{k}\right\}_{k=1}^{N_{0}}$. The above convergence implies that $v_{N} \rightarrow v, f_{N} \rightarrow f$ also in $C\left(0, T_{0} ; L^{2}\left(\mathbb{T}^{d}\right)\right)$. Therefore, the the initial and terminal conditions on $f, v$ hold as well. Since $\cup_{N \geq 1} \operatorname{span}\left\{e_{k}\right\}_{k=1}^{N}$ is dense in $L^{2}\left(0, T_{0} ; H^{1}\left(\mathbb{T}^{d}\right)\right)$, we are done.

Theorem 8 (Higher Regularity). Let $\left(u_{\lambda}, m_{\lambda}\right)$ be a $C^{\infty}$ solution of (20) and let $T_{0}$ be as in Theorem 4. Assume $A, B \in H^{k+1}\left(\mathbb{T}^{d}\right), h, g \in F^{2 k}\left(\mathcal{T} ; \mathbb{T}^{d}\right)$ and let $W=[h, g, A, B]^{t}$. Then, under Assumptions 11 12, for any weak solution $w=[f, v]^{t}$ of $\mathcal{L}_{\lambda} w=W$, we have $v, f \in F^{2 k+2}\left(\mathcal{T} ; \mathbb{T}^{d}\right)$.

The proof draws on the regularizing properties of the heat equation and a bootstrap argument. We use the following result: 
Lemma 6. Let $\tilde{h} \in H^{k_{1}}\left(\mathcal{T} ; H^{k_{2}}\left(\mathbb{T}^{d}\right)\right), \tilde{g} \in H^{2 k_{1}+k_{2}+1}\left(\mathbb{T}^{d}\right)$ for some $k_{1}, k_{2} \geq 0$, and let $\tilde{u} \in$ $F^{1}\left(\mathcal{T} ; \mathbb{T}^{d}\right)$ be a weak solution of the heat equation

$$
\left\{\begin{array}{l}
\tilde{u}_{t}-\Delta \tilde{u}=\tilde{h} \\
\tilde{u}(x, 0)=\tilde{g}(x) .
\end{array}\right.
$$

Then $\tilde{u} \in H^{k_{1}}\left(\mathcal{T} ; H^{k_{2}+2}\left(\mathbb{T}^{d}\right)\right) \cap H^{k_{1}+1}\left(\mathcal{T} ; H^{k_{2}}\left(\mathbb{T}^{d}\right)\right)$.

Proof. The Lemma is proved easily using induction. The base case $k_{1}=k_{2}=0$ is a standard regularity result for the heat equation.

From the second equation of (23), we have that $v$ is a weak solution to (30)

$$
\left\{\begin{array}{l}
v_{t}+\Delta v=g \\
\quad+\alpha m^{\alpha-1} f\left(H_{\lambda}\left(x, \frac{D u}{m^{\alpha}}\right)+\frac{D u}{m^{\alpha}} D_{p} H_{\lambda}\left(x, \frac{D u}{m^{\alpha}}\right)\right)+D_{p} H_{\lambda}\left(x, \frac{D u}{m^{\alpha}}\right) D v+b_{\lambda} \cdot D v+D_{z} V_{\lambda} f \\
v\left(x, T_{0}\right)=B(x) .
\end{array}\right.
$$

Since the right-hand side of the previous PDE belongs to $L^{2}\left(0, T_{0}, L^{2}\left(\mathbb{T}^{d}\right)\right)$, using Lemma 6 , we conclude that $v \in L^{2}\left(\mathcal{T} ; H^{2}\left(\mathbb{T}^{d}\right)\right) \cap H^{1}\left(\mathcal{T} ; L^{2}\left(\mathbb{T}^{d}\right)\right)$.

Next, the first equation of (23) implies that $f$ is a weak solution to

$$
\left\{\begin{array}{l}
f_{t}-\Delta f=h \\
\quad+\operatorname{div}\left[D_{p} H_{\lambda}\left(x, \frac{D u}{m^{\alpha}}\right) f-\alpha f D_{p p}^{2} H_{\lambda}\left(x, \frac{D u}{m^{\alpha}}\right) \cdot \frac{D u}{m^{\alpha}}+m^{1-\alpha} D_{p p}^{2} H_{\lambda}\left(x, \frac{D u}{m^{\alpha}}\right) \cdot D v+b_{\lambda} f\right] \\
f(x, 0)=A(x) .
\end{array}\right.
$$

From the regularity of $v$ obtained above, we conclude that the right-hand side of this equation is also in $L^{2}\left(0, T_{0}, L^{2}\left(\mathbb{T}^{d}\right)\right)$. For that reason, according to Lemma $6 f \in L^{2}\left(\mathcal{T} ; H^{2}\left(\mathbb{T}^{d}\right)\right) \cap$ $H^{1}\left(\mathcal{T} ; L^{2}\left(\mathbb{T}^{d}\right)\right)$.

Now, we assume $v, f \in F^{2 i}\left(\mathcal{T} ; \mathbb{T}^{d}\right)$ for some $i \leq k$, we will prove that $v, f \in F^{2 i+2}\left(\mathcal{T} ; \mathbb{T}^{d}\right)$. First, note that since $v, f \in H^{k_{1}}\left(\mathcal{T} ; H^{k_{2}}\left(\mathbb{T}^{d}\right)\right)$ for every $k_{1}, k_{2}$ with $2 k_{1}+k_{2}=2 i$, the expression on the right-hand side of (30) is in $H^{k_{1}}\left(\mathcal{T} ; H^{k_{2}-1}\left(\mathbb{T}^{d}\right)\right)$. Thus, using Lemma 6 we get $v \in$ $H^{k_{1}}\left(\mathcal{T} ; H^{k_{2}+1}\left(\mathbb{T}^{d}\right)\right)$. We know now that the right-hand side of $(30)$ is in $H^{k_{1}}\left(\mathcal{T} ; H^{k_{2}}\left(\mathbb{T}^{d}\right)\right)$. Using Lemma [6 the second time, we conclude that $v \in H^{k_{1}}\left(\mathcal{T} ; H^{k_{2}+2}\left(\mathbb{T}^{d}\right)\right) \cap H^{k_{1}+1}\left(\mathcal{T} ; H^{k_{2}}\left(\mathbb{T}^{d}\right)\right)$.

Now, we have that the right-hand side of (31) is in $H^{k_{1}}\left(\mathcal{T} ; H^{k_{2}-1}\left(\mathbb{T}^{d}\right)\right)$. Thus, using Lemma 6 again twice as above, we get $f \in H^{k_{1}}\left(\mathcal{T} ; H^{k_{2}+2}\left(\mathbb{T}^{d}\right)\right) \cap H^{k_{1}+1}\left(\mathcal{T} ; H^{k_{2}}\left(\mathbb{T}^{d}\right)\right)$. From what we have proved, it follows $v, f \in H^{\tilde{k}_{1}}\left(\mathcal{T} ; H^{\tilde{k}_{2}}\left(\mathbb{T}^{d}\right)\right)$, for every $\tilde{k}_{1}, \tilde{k}_{2}$ with $2 \tilde{k}_{1}+\tilde{k}_{2}=2 i+2$. Consequently, $v, f \in F^{i+2}\left(\mathcal{T} ; \mathbb{T}^{d}\right)$.

Proof of the Theorem 1 . Theorem 4 and Arzela-Ascoli Theorem imply that the set $\Lambda$ is a closed subset of the interval $[0,1]$. We will prove that it is also open. Let $\lambda_{0} \in \Lambda$. Using Theorem 4, we see that the operator

$$
\mathcal{L}_{\lambda_{0}}: F^{2 k}\left(\mathcal{T} ; \mathbb{T}^{d}\right) \times F^{2 k}\left(\mathcal{T} ; \mathbb{T}^{d}\right) \rightarrow F^{2 k-2}\left(\mathcal{T} ; \mathbb{T}^{d}\right) \times F^{2 k-2}\left(\mathcal{T} ; \mathbb{T}^{d}\right) \times H^{2 k-1}\left(\mathbb{T}^{d}\right) \times H^{2 k-1}\left(\mathbb{T}^{d}\right)
$$

is bounded for every $k \geq 1$. Using Theorems 5 , 7 and 8 we conclude that $\mathcal{L}_{\lambda_{0}}$ is bijective, and so it is invertible. We choose $k$ large enough so that $H^{l}\left(\mathcal{T} ; H^{l}\left(\mathbb{T}^{d}\right)\right)$, where $l=\left\lfloor\frac{2 k}{3}\right\rfloor$, is an algebra. By the inverse function theorem ([3]), there is a neighborhood $U$ of $\lambda_{0}$ where the equation $\mathcal{M}_{\lambda}\left[\begin{array}{c}u \\ m\end{array}\right]=0$ has a unique solution $\left(u_{\lambda}, m_{\lambda}\right)$ in $F^{2 k}\left(\mathcal{T} ; \mathbb{T}^{d}\right) \times F^{2 k}\left(\mathcal{T} ; \mathbb{T}^{d}\right)$. Then, $u_{\lambda}, m_{\lambda} \in H^{l}\left(\mathcal{T} ; H^{l}\left(\mathbb{T}^{d}\right)\right)$. The inverse function theorem implies that the mapping $\lambda \mapsto\left(u_{\lambda}, m_{\lambda}\right)$ is continuous. Hence, we can assume that in the neighborhood $U, m_{\lambda}$ is bounded away from zero. This observation, together with the fact that $H^{l}\left(\mathcal{T} ; H^{l}\left(\mathbb{T}^{d}\right)\right)$ is an algebra allows us to use regularity theory and bootstrap arguments to conclude that $\left(u_{\lambda}, m_{\lambda}\right)$ are $C^{\infty}$. Accordingly, 
$U \subset \Lambda$. Consequently, we have proved that $\Lambda$ is an open set in $[0,1]$. Because $1 \in \Lambda$, we know that $\Lambda \neq \emptyset$. Therefore, $\Lambda=[0,1]$. In particular, $0 \in \Lambda$.

\section{REFERENCES}

[1] M. Burger, M. Di Francesco, P. A. Markowich, and M.-T. Wolfram. Mean field games with nonlinear mobilities in pedestrian dynamics. Discrete Contin. Dyn. Syst. Ser. B, 19(5):1311-1333, 2014.

[2] P. Cardaliaguet, P. Garber, A. Porretta, and D. Tonon. Second order mean field games with degenerate diffusion and local coupling. Preprint, 2014.

[3] J. Dieudonné. Foundations of modern analysis. Vol. I, Academic Press, New York, 1969.

[4] L. C. Evans. Partial Differential Equations. Graduate Studies in Mathematics. American Mathematical Society, 1998.

[5] L. C. Evans. Adjoint and compensated compactness methods for Hamilton-Jacobi PDE. Arch. Ration. Mech. Anal., 197(3):1053-1088, 2010.

[6] R. Ferreira and D. Gomes. On the convergence of finite state mean-field games through $\Gamma$-convergence. J. Math. Anal. Appl., 418(1):211-230, 2014.

[7] D. Gomes. Continuous time finite state space mean field games - a variational approach. 2011 49th Annual Allerton Conference on Communication, Control, and Computing, Allerton 2011, pages 998-1001, 2011.

[8] D. Gomes and H. Mitake. Stationary mean-field games with congestion and quadratic Hamiltonians. Preprint.

[9] D. Gomes, J. Mohr, and R. R. Souza. Discrete time, finite state space mean field games. Journal de Mathématiques Pures et Appliquées, 93(2):308-328, 2010.

[10] D. Gomes, J. Mohr, and R. R. Souza. Continuous time finite state mean-field games. Appl. Math. and Opt., 68(1):99-143, 2013.

[11] D. Gomes and S. Patrizi. Obstacle mean-field game problem. To appear in Interfaces and Free Boundaries, 2013.

[12] D. Gomes, S. Patrizi, and V. Voskanyan. On the existence of classical solutions for stationary extended mean field games. Nonlinear Anal., 99:49-79, 2014.

[13] D. Gomes and E. Pimentel. Local regularity for mean-field games in the whole space. Preprint.

[14] D. Gomes and E. Pimentel. Time dependent mean-field games with logarithmic nonlinearities. Preprint.

[15] D. Gomes, E. Pimentel, and H Sanchez-Morgado. Time dependent mean-field games in the superquadratic case. Preprint, 2013.

[16] D. Gomes, E. A. Pimentel, and H. Sánchez-Morgado. Time-dependent mean-field games in the subquadratic case. Comm. Partial Differential Equations, 40(1):40-76, 2015.

[17] D. Gomes, G. E. Pires, and H. Sánchez-Morgado. A-priori estimates for stationary mean-field games. Netw. Heterog. Media, 7(2):303-314, 2012.

[18] D. Gomes and H. Sánchez Morgado. A stochastic Evans-Aronsson problem. Trans. Amer. Math. Soc., 366(2):903-929, 2014

[19] D. Gomes and J. Saúde. Mean field games models - a brief survey. Dyn. Games Appl., 4(2):110-154, 2014.

[20] D. Gomes, R. M. Velho, and M.-T. Wolfram. Dual two-state mean-field games. Proceedings CDC $2014,2014$.

[21] D. Gomes, R. M. Velho, and Marie-Therese Wolfram. Socio-economic applications of finite state mean field games. Philos. Trans. R. Soc. Lond. Ser. A Math. Phys. Eng. Sci., 372(2028):20130405, $18,2014$.

[22] D. Gomes and V. Voskanyan. Extended deterministic mean-field games. Preprint, 2013.

[23] J. Graber. Weak solutions for mean field games with congestion. Preprint.

[24] O. Guéant. A uniqueness result for mean field games. Classnotes, 2011.

[25] M. Huang, P. E. Caines, and R. P. Malhamé. Large-population cost-coupled LQG problems with nonuniform agents: individual-mass behavior and decentralized $\epsilon$-Nash equilibria. IEEE Trans. Automat. Control, 52(9):1560-1571, 2007.

[26] M. Huang, R. P. Malhamé, and P. E. Caines. Large population stochastic dynamic games: closed-loop McKeanVlasov systems and the Nash certainty equivalence principle. Commun. Inf. Syst., 6(3):221-251, 2006.

[27] O.A. Ladyzenskaja, V.A. Solonnikov, and Ural'ceva N.N. Linear and quasilinear equations of pbolic type., volume 27 of Translations of Mathematical Monographs. American Mathematical Society, Providence, RI, 1967.

[28] J.-M. Lasry and P.-L. Lions. Jeux à champ moyen. I. Le cas stationnaire. C. R. Math. Acad. Sci. Paris, 343(9):619-625, 2006.

[29] J.-M. Lasry and P.-L. Lions. Jeux à champ moyen. II. Horizon fini et contrôle optimal. C. R. Math. Acad. Sci. Paris, 343(10):679-684, 2006.

[30] J.-M. Lasry and P.-L. Lions. Mean field games. Jpn. J. Math., 2(1):229-260, 2007.

[31] P.-L. Lions. College de France course on mean-field games. 2007-2011.

[32] A. R. Mészáros and F. J. Silva. A variational approach to second order mean field games with density constraints: the stationary case. arXiv preprint arXiv:1502.06026, 2015.

[33] A. Porretta. On the planning problem for the mean-field games system. Dyn. Games Appl., 2013. 
[34] A. Porretta. Weak Solutions to Fokker-Planck Equations and Mean Field Games. Arch. Ration. Mech. Anal., 216(1):1-62, 2015.

[35] F. Santambrogio. A modest proposal for MFG with density constraints. Netw. Heterog. Media, 7(2):337-347, 2012.

[36] H. V. Tran. Adjoint methods for static Hamilton-Jacobi equations. Calculus of Variations and PDE, 41:301$319,2011$.

(D. A. Gomes) King Abdullah University of Science and Technology (KAUST), CSmSe Division , Thuwal 23955-6900. Saudi Arabia, and KAUSt SRI, Uncertainty Quantification Center in ComputaTiOnal SCIENCE ANd EngineERING.

E-mail address: diogo.gomes@kaust.edu.sa

(V. Voskanyan) King Abdullah University of Science and Technology (KAUST), CSmSE Division , Thuwal 23955-6900. Saudi Arabia, and KAUSt SRI, Uncertainty Quantification Center in ComputaTiONAL SCIENCE AND ENGINEERING.

E-mail address: vardan.voskanyan@kaust.edu.sa 